July 1998

\title{
3-point Functions in Conformal Field Theory with Affine Lie Group Symmetry
}

\author{
Jørgen Rasmussen円 \\ Laboratoire de Mathématiques et Physique Théorique, \\ Université de Tours, Parc de Grandmont, F-37200 Tours, France
}

\begin{abstract}
In this paper we develop a general method for constructing 3-point functions in conformal field theory with affine Lie group symmetry, continuing our recent work on 2-point functions. The results are provided in terms of triangular coordinates used in a wave function description of vectors in highest weight modules. In this framework, complicated couplings translate into ordinary products of certain elementary polynomials. The discussions pertain to all simple Lie groups and arbitrary integrable representation. An interesting by-product is a general procedure for computing tensor product coefficients, essentially by counting integer solutions to certain inequalities. As an illustration of the construction, we consider in great detail the three cases $S L(3), S L(4)$ and $S O(5)$.
\end{abstract}

PACS: $11.25 . \mathrm{Hf}$

Keywords: Conformal field theory; affine current algebra, correlation functions

\footnotetext{
${ }^{1}$ e-mail address: jorgen@celfi.phys.univ-tours.fr
} 


\section{Introduction}

The work presented in this paper constitutes a continuation of our recent work on 2-point functions in conformal field theory with affine Lie group symmetry or affine Lie algebra for short [1]. Our aim is to develop a systematic and general approach to the computation of correlators. Thus, in [1] the program was initiated by considering 2-point functions for all Lie groups and arbitrary representations, integrable or non-integrable. In this paper we undertake the study of 3-point functions for integrable representations and present modest speculations on a possible generalization to non-integrable representations.

Our method is based on a wave function formalism where vectors in certain representation spaces are translated into polynomials in so-called triangular coordinates $x$. In this framework, complicated couplings of highest weight representations are described by ordinary products of the polynomials. We shall use the notion of elementary couplings known from the study of tensor or Kronecker products of integrable highest weight representations, see e.g. [2, 3, 4] and references therein. This gives rise to a finite set of elementary polynomials in terms of which our results may be expressed. Certain redundancies, known as syzygies, appear in our formalism as vanishing linear combinations of simple products of these elementary polynomials.

To the best of our knowledge, the only systematic approach to 3-point functions in the literature is the work [5] by Rühl]. However, his construction is designed explicitly to treat integrable representations in the case of $S L(N)$ only. In Section 4 we shall make some comparative comments.

It should be stressed that our results are completely general wrt the Lie algebra and the integrable representations, but pertain to the global or horizontal structure of the 3 -point function. It is the dependence on the affine coordinates $x$ that is determined in addition to the well known dependence on the conformal coordinates $z$. Thus, we do not discuss the role played by the central extension (or level) $k$ of the affine Lie algebra. Information on that is encoded in the structure constants which are yet to be determined. They are closely related to the physical fusion coefficients. In the classical limit $k \rightarrow \infty$ these reduce to the ordinary tensor product coefficients. By construction, the latter denote the dimensionalities of the spaces of chiral blocks that we obtain. Thus, in the process of constructing the complete and minimal solution spaces for the 3-point functions we are also determining the tensor product coefficients. This by-product is an alternative to more conventional methods.

Besides providing new and systematic results on 3-point functions, our construction seems amenable of generalizing to non-integrable representations. The indication is found in the very simple mathematical structure of the correlators, where only linear combinations of ordinary products of polynomials appear. As discussed in Section 5, a generalization is currently under investigation.

The remaining part of this paper is organized as follows. In Section 2 we present some background material. In Section 3 we introduce the wave function formalism and discuss the general structure of 3-point functions in terms of elementary polynomials implementing the syzygies. As building blocks, we introduce generalized anharmonic ratios. In Section 4 we discuss the cases $S L(3), S O(5)$ and $S L(4)$ and present some

\footnotetext{
${ }^{1}$ We thank P. Furlan, A.Ch. Ganchev and V.B. Petkova for pointing out this work.
} 
explicit results working out examples. Section 5 contains some concluding speculations on possible generalizations, while various technical results on $S O(5)$ and $S L(4)$ are listed in Appendix A.

\section{Notation}

Let $\mathbf{g}$ be a simple Lie algebra of rank $r$. $\mathbf{h}$ is a Cartan subalgebra of $\mathbf{g}$. The set of (positive) roots is denoted $\left(\Delta_{+}\right) \Delta$ and the simple roots are written $\alpha_{i}, i=1, \ldots, r$. $\alpha^{\vee}=2 \alpha / \alpha^{2}$ is the root dual to $\alpha$. Using the triangular decomposition

$$
\mathbf{g}=\mathbf{g}_{-} \oplus \mathbf{h} \oplus \mathbf{g}_{+}
$$

the raising and lowering operators are denoted $e_{\alpha} \in \mathbf{g}_{+}$and $f_{\alpha} \in \mathbf{g}_{-}$, respectively, with $\alpha \in \Delta_{+}$, and $h_{i} \in \mathbf{h}$ are the Cartan operators. In the Cartan-Weyl basis we have

$$
\left[h_{i}, e_{\alpha}\right]=\left(\alpha_{i}^{\vee}, \alpha\right) e_{\alpha} \quad, \quad\left[h_{i}, f_{\alpha}\right]=-\left(\alpha_{i}^{\vee}, \alpha\right) f_{\alpha}
$$

and

$$
\left[e_{\alpha}, f_{\alpha}\right]=h_{\alpha}=G^{i j}\left(\alpha_{i}^{\vee}, \alpha^{\vee}\right) h_{j}
$$

where the metric $G_{i j}$ is related to the Cartan matrix $A_{i j}$ as

$$
A_{i j}=\alpha_{i}^{\vee} \cdot \alpha_{j}=\left(\alpha_{i}^{\vee}, \alpha_{j}\right)=G_{i j} \alpha_{j}^{2} / 2
$$

The Dynkin labels $\Lambda_{k}$ of the weight $\Lambda$ are defined by

$$
\Lambda=\Lambda_{k} \Lambda^{k} \quad, \quad \Lambda_{k}=\left(\alpha_{k}^{\vee}, \Lambda\right)
$$

where $\left\{\Lambda^{k}\right\}_{k=1, \ldots, r}$ is the set of fundamental weights satisfying

$$
\left(\alpha_{i}^{\vee}, \Lambda^{k}\right)=\delta_{i}^{k}
$$

Elements in $\mathbf{g}_{+}$may be parameterized using "triangular coordinates" denoted by $x^{\alpha}$, one for each positive root, thus we write general Lie algebra elements in $\mathbf{g}_{+}$as

$$
g_{+}(x)=x^{\alpha} e_{\alpha} \in \mathbf{g}_{+}
$$

We will understand "properly" repeated root indices as in (7) to be summed over the positive roots. Repeated Cartan indices as in (5) are also summed over. The corresponding group element is

$$
G_{+}(x)=e^{g_{+}(x)}
$$

The matrix representation $C(x)$ of $g_{+}(x)$ in the adjoint representation is defined by

$$
C_{a}^{b}(x)=-x^{\beta} f_{\beta a}^{b}
$$

Now, a differential operator realization $\left\{\tilde{J}_{a}(x, \partial, \Lambda)\right\}$ of the simple Lie algebra g generated by $\left\{j_{a}\right\}$ may be defined by

$$
\langle\Lambda, x| j_{a}=\tilde{J}_{a}(x, \partial, \Lambda)\langle\Lambda, x|
$$


where

$$
\langle\Lambda, x|=\langle\Lambda| G_{+}(x)
$$

The explicit solution is found to be [6]

$$
\begin{aligned}
\tilde{E}_{\alpha}(x, \partial) & =V_{\alpha}^{\beta}(x) \partial_{\beta} \\
\tilde{H}_{i}(x, \partial, \Lambda) & =V_{i}^{\beta}(x) \partial_{\beta}+\Lambda_{i} \\
\tilde{F}_{\alpha}(x, \partial, \Lambda) & =V_{-\alpha}^{\beta}(x) \partial_{\beta}+P_{-\alpha}^{j}(x) \Lambda_{j}
\end{aligned}
$$

where

$$
\begin{aligned}
V_{\alpha}^{\beta}(x) & =[B(C(x))]_{\alpha}^{\beta} \\
V_{i}^{\beta}(x) & =-[C(x)]_{i}^{\beta} \\
V_{-\alpha}^{\beta}(x) & =\left[e^{-C(x)}\right]_{-\alpha}^{\gamma}[B(-C(x))]_{\gamma}^{\beta} \\
P_{-\alpha}^{j}(x) & =\left[e^{-C(x)}\right]_{-\alpha}^{j}
\end{aligned}
$$

$B$ is the generating function for the Bernoulli numbers

$$
B(u)=\frac{u}{e^{u}-1}=\sum_{n \geq 0} \frac{B_{n}}{n !} u^{n}
$$

whereas $\partial_{\beta}$ denotes partial differentiation wrt $x^{\beta}$. Closely related to this differential operator realization is the equivalent one $\left\{J_{a}(x, \partial, \Lambda)\right\}$ given by

$$
\begin{aligned}
E_{\alpha}(x, \partial, \Lambda) & =-\tilde{F}_{\alpha}(x, \partial, \Lambda) \\
F_{\alpha}(x, \partial, \Lambda) & =-\tilde{E}_{\alpha}(x, \partial, \Lambda) \\
H_{i}(x, \partial, \Lambda) & =-\tilde{H}_{i}(x, \partial, \Lambda)
\end{aligned}
$$

The matrix functions (13) are defined in terms of universal power series expansions, valid for any Lie algebra, but ones that truncate giving rise to finite polynomials of which the explicit forms depend on the Lie algebra under consideration. Details on the truncations and the resulting polynomials may be found in [6].

\subsection{Affine Current Algebra}

Associated to a Lie algebra is an affine Lie algebra characterized by the central extension

$k$, and associated to an affine Lie algebra is an affine current algebra whose generators are conformal spin one fields and have amongst themselves the operator product expansion

$$
J_{a}(z) J_{b}(w)=\frac{\kappa_{a b} k}{(z-w)^{2}}+\frac{f_{a b}^{c} J_{c}(w)}{z-w}
$$

where regular terms have been omitted. $\kappa_{a b}$ and $f_{a b}{ }^{c}$ are the Cartan-Killing form and the structure coefficients, respectively, of the underlying Lie algebra. 
It is convenient to collect the traditional multiplet of primary fields in an affine current algebra (which generically is infinite for non-integrable representations) in a generating function for that [0, 8, 6], namely the primary field $\phi_{\Lambda}(w, x)$ which must satisfy

$$
\begin{aligned}
J_{a}(z) \phi_{\Lambda}(w, x) & =\frac{-J_{a}(x, \partial, \Lambda)}{z-w} \phi_{\Lambda}(w, x) \\
T(z) \phi_{\Lambda}(w, x) & =\frac{\Delta\left(\phi_{\Lambda}\right)}{(z-w)^{2}} \phi_{\Lambda}(w, x)+\frac{1}{z-w} \partial \phi_{\Lambda}(w, x)
\end{aligned}
$$

Here $\left\{J_{a}(z)\right\}$ and $T(z)$ are the affine currents and the energy-momentum tensor, respectively, whereas $\left\{J_{a}(x, \partial, \Lambda)\right\}$ is the differential operator realization. $\Delta\left(\phi_{\Lambda}\right)$ denotes the conformal dimension of $\phi_{\Lambda}$. The explicit construction of primary fields for general simple Lie algebra and arbitrary representation is provided in [6], see also the discussion on wave functions below.

An affine transformation of a primary field is given by

$$
\begin{aligned}
\delta_{\epsilon} \phi_{\Lambda}(w, x) & =\oint_{w} \frac{d z}{2 \pi i} \epsilon^{a}(z) J_{a}(z) \phi_{\Lambda}(w, x) \\
& =\left\{\epsilon^{-\alpha}(w) V_{\alpha}^{\beta}(x) \partial_{\beta}+\epsilon^{i}(w)\left(V_{i}^{\beta}(x) \partial_{\beta}+\Lambda_{i}\right)\right. \\
& \left.+\epsilon^{\alpha}(w)\left(V_{-\alpha}^{\beta}(x) \partial_{\beta}+P_{-\alpha}^{i}(x) \Lambda_{i}\right)\right\} \phi_{\Lambda}(w, x)
\end{aligned}
$$

and is parameterized by the $d$ ( $d$ is the dimension of the underlying Lie algebra) independent infinitesimal functions $\epsilon^{a}(z)$.

\section{3-point Functions}

Let $W_{3}\left(z_{1}, z_{2}, z_{3} ; x_{1}, x_{2}, x_{3} ; \Lambda_{(1)}, \Lambda_{(2)}, \Lambda_{(3)} ; k\right)$ denote a general 3 -point function of the 3 primary fields $\phi_{\Lambda_{(1)}}\left(z_{1}, x_{1}\right), \phi_{\Lambda_{(2)}}\left(z_{2}, x_{2}\right)$ and $\phi_{\Lambda_{(3)}}\left(z_{3}, x_{3}\right)$. Recall that $k$ is the central extension. The conformal Ward identities or projective invariance allow us to determine completely the conformal property ( $z$ dependence) of the 3 -point function, whereby we may write

$$
\begin{aligned}
& W_{3}\left(z_{1}, z_{2}, z_{3} ; x_{1}, x_{2}, x_{3} ; \Lambda_{(1)}, \Lambda_{(2)}, \Lambda_{(3)} ; k\right) \\
= & C_{\Lambda_{(1)} \Lambda_{(2)} \Lambda_{(3)}}(k)\left(z_{1}-z_{2}\right)^{-\Delta_{1}-\Delta_{2}+\Delta_{3}}\left(z_{2}-z_{3}\right)^{-\Delta_{2}-\Delta_{3}+\Delta_{1}}\left(z_{3}-z_{1}\right)^{-\Delta_{3}-\Delta_{1}+\Delta_{2}} \\
& W_{n}^{\text {aff }}\left(x_{1}, x_{2}, x_{3} ; \Lambda_{(1)}, \Lambda_{(2)}, \Lambda_{(3)}\right)
\end{aligned}
$$

where $\Delta_{l}=\Delta\left(\phi_{\Lambda_{(l)}}\right)$ is the conformal weight of the primary field $\phi_{\Lambda_{(l)}}$. The affine Ward identity

$$
\begin{aligned}
0 & =\delta_{\epsilon} W_{3}\left(z_{1}, z_{2}, z_{3} ; x_{1}, x_{2}, x_{3} ; \Lambda_{(1)}, \Lambda_{(2)}, \Lambda_{(3)} ; k\right) \\
& =\left\langle\delta_{\epsilon} \phi_{\Lambda_{(1)}}\left(z_{1}, x_{1}\right) \phi_{\Lambda_{(2)}}\left(z_{2}, x_{2}\right) \phi_{\Lambda_{(3)}}\left(z_{3}, x_{3}\right)\right\rangle+\left\langle\phi_{\Lambda_{(1)}}\left(z_{1}, x_{1}\right) \delta_{\epsilon} \phi_{\Lambda_{(2)}}\left(z_{2}, x_{2}\right) \phi_{\Lambda_{(3)}}\left(z_{3}, x_{3}\right)\right\rangle \\
& +\left\langle\phi_{\Lambda_{(1)}}\left(z_{1}, x_{1}\right) \phi_{\Lambda_{(2)}}\left(z_{2}, x_{2}\right) \delta_{\epsilon} \phi_{\Lambda_{(3)}}\left(z_{3}, x_{3}\right)\right\rangle
\end{aligned}
$$

may then be recast (using (18)) into the following set of $d$ partial differential equations

$$
\left(\sum_{l=1}^{3} \tilde{J}_{a}\left(x_{l}, \partial, \Lambda_{(l)}\right)\right) W_{3}^{a f f}\left(x_{1}, x_{2}, x_{3} ; \Lambda_{(1)}, \Lambda_{(2)}, \Lambda_{(3)}\right)=0
$$


It is easily verified that only the $2 r$ equations for $a= \pm \alpha_{i}$ are independent. By induction, this simply follows from the fact that $\left\{\tilde{J}_{a}\right\}$ is a differential operator realization of a Lie algebra. It is the (finite dimensional) solution spaces to the equations (21) for integrable representations that we shall discuss in the following. We shall use the notation $W_{3}^{a f f, m}$ to indicate the multiplicities. Thus, $m=1, \ldots, N_{\Lambda_{(1)} \Lambda_{(2)} \Lambda_{(3)}}$ where $N_{\Lambda_{(1)} \Lambda_{(2)} \Lambda_{(3)}}$ is the tensor product coefficient for the coupling $\left(\Lambda_{(1)}, \Lambda_{(2)}, \Lambda_{(3)}\right)$. Note that we are only discussing the global ( $k$ independent) behavior of the correlators, so $N_{\Lambda_{(1)} \Lambda_{(2)} \Lambda_{(3)}}$ is also the dimension of the space of chiral blocks for the given coupling. The non-trivial dependence on the central extension $k$ is encoded in the coupling constants $C_{\Lambda_{(1)} \Lambda_{(2)} \Lambda_{(3)}}(k)$. In general, also the dimension of the space of physical chiral blocks will depend on $k$ since it is given by the fusion coefficient $N_{\Lambda_{(1)} \Lambda_{(2)} \Lambda_{(3)}}(k)$ (see e.g. [3, [4] and [9]) satisfying

$$
N_{\Lambda_{(1)} \Lambda_{(2)} \Lambda_{(3)}}(k) \leq N_{\Lambda_{(1)} \Lambda_{(2)} \Lambda_{(3)}}(k=\infty)=N_{\Lambda_{(1)} \Lambda_{(2)} \Lambda_{(3)}}
$$

\subsection{Wave Functions}

Here we shall discuss a wave function picture of the Kronecker or tensor product of a set of integrable highest weight representations. The idea [6, 1] is to translate states or vectors in the representation spaces into polynomials in the triangular coordinates $x$, upon which vectors in the tensor product modules are given by ordinary products of polynomials. First we review a few basic properties of conjugate weights and fundamental representations.

In every highest weight representation of highest weight $\Lambda$ the weights are given by $\lambda=\Lambda-\sum \beta$ where $\sum \beta$ is a sum of positive roots or zero. The depth of $\lambda$ is then defined as the height of $\sum \beta$. In a finite dimensional irreducible highest weight module there exists a unique vector (up to trivial renormalizations) of lowest weight characterized by having maximal depth. The conjugate representation of such a representation is a highest weight representation with highest weight $\Lambda^{+}$given by minus the lowest weight of the original one, while in general all weights in the conjugate representation are given by minus the ones in the original representation. The conjugate representation of a fundamental representation (which is a finite dimensional irreducible highest weight representation of highest weight a fundamental weight) is again a fundamental representation. Due to the uniqueness of the conjugate weight we shall write $\Lambda^{i^{+}}=\left(\Lambda^{i}\right)^{+}$. Many fundamental representations (and therefore also many non-fundamental representations) are self-conjugate, see e.g. [10]. In [1], the natural generalization of the notion of conjugate weight to non-integrable representations is discussed and the obvious result is that the conjugate weight $\Lambda^{+}$to an arbitrary weight $\Lambda=\sum_{k=1}^{r} \Lambda_{k} \Lambda^{k}$, integrable or non-integrable, is given by

$$
\Lambda^{+}=\sum_{k=1}^{r} \Lambda_{k}^{+} \Lambda^{k}=\sum_{k=1}^{r} \Lambda_{k^{+}} \Lambda^{k}
$$

Let $\Lambda$ be an arbitrary integrable weight and let $\{|\lambda\rangle\}^{\mu}$ denote a (not necessarily orthonormal) basis in the highest weight module generated from the highest weight vector $|\Lambda\rangle$. A basis element may generically be written

$$
|\lambda\rangle^{\mu}=f_{\alpha_{j_{1}}} \ldots f_{\alpha_{j_{n}(\mu)}}|\Lambda\rangle
$$


The corresponding wave function is defined by

$$
\begin{aligned}
b\left(x, \Lambda,\left\{j_{l}\right\}^{\mu}\right) & =\langle\Lambda, x \mid \lambda\rangle^{\mu} \\
& =\tilde{F}_{\alpha_{j_{1}}}(x, \partial, \Lambda) \ldots \tilde{F}_{\alpha_{j_{n}(\mu)}}(x, \partial, \Lambda) 1 \\
& =\left(V_{-\alpha_{j_{1}}}^{\beta}(x) \partial_{\beta}+P_{-\alpha_{j_{1}}}^{i_{1}}(x) \Lambda_{i_{1}}\right) \ldots \\
& \cdot\left(V_{-\alpha_{j_{n(\mu)-1}}}^{\beta}(x) \partial_{\beta}+P_{-\alpha_{j_{n}(\mu)-1}}^{i_{n(\mu)-1}}(x) \Lambda_{i_{n(\mu)-1}}\right) P_{-\alpha_{j_{n(\mu)}}}^{i_{n(\mu)}}(x) \Lambda_{i_{n(\mu)}}
\end{aligned}
$$

Wave functions for elements in the tensor product of a set of integrable highest weight modules $\left(\Lambda_{(1)}, \ldots, \Lambda_{(n)}\right)$ are then simply given by finite sums of products of wave functions

$$
\begin{aligned}
& \sum_{\mu_{1}, \ldots, \mu_{n}} C_{\mu_{1} \ldots \mu_{n}}\left|\lambda_{(1)}\right\rangle^{\mu_{1}} \otimes \ldots \otimes\left|\lambda_{(n)}\right\rangle^{\mu_{n}} \\
\rightarrow & \left\langle\Lambda_{(1)}, x_{1}\left|\otimes \ldots \otimes\left\langle\Lambda_{(n)}, x_{n}\left|\sum_{\mu_{1}, \ldots, \mu_{n}} C_{\mu_{1} \ldots \mu_{n}}\right| \lambda_{(1)}\right\rangle^{\mu_{1}} \otimes \ldots \otimes\right| \lambda_{(n)}\right\rangle^{\mu_{n}} \\
= & \sum_{\mu_{1}, \ldots, \mu_{n}} C_{\mu_{1} \ldots \mu_{n}} b\left(x_{1}, \Lambda_{(1)},\left\{j_{l_{1}}\right\}^{\mu_{1}}\right) \ldots b\left(x_{n}, \Lambda_{(n)},\left\{j_{l_{n}}\right\}^{\mu_{n}}\right)
\end{aligned}
$$

\subsection{Elementary Couplings and Polynomials}

When considering generating functions for tensor product coefficients and branching rule multiplicities, fusion coefficients etc, elementary couplings are very useful, see [2, 3, 国 and references therein. This is due to their basis properties: they are linearly independent, all other couplings may be expressed in terms of them while they cannot themselves be expressed in terms of other (elementary) couplings. In the following we shall be interested mainly in 3-point couplings to the scalar representation, though the notation introduced here allows for discussion of general $n$-point couplings.

Let $\mathcal{L}_{n}^{l}$ denote the set of elementary $n$-point couplings involving the identity or zero weight exactly $n-l$ times. The set of associated elementary polynomials (corresponding wave functions) are then denoted $\mathcal{E}_{n}^{l}$. The total sets are denoted

$$
\mathcal{L}_{n}=\bigcup_{l=2}^{n} \mathcal{L}_{n}^{l} \quad, \quad \mathcal{E}_{n}=\bigcup_{l=2}^{n} \mathcal{E}_{n}^{l}
$$

The element in $\mathcal{E}_{n}$ associated to the coupling $\left(\lambda_{(1)}, \ldots, \lambda_{(n)}\right) \in \mathcal{L}_{n}$ is denoted

$$
R^{\lambda_{(1)}, \ldots, \lambda_{(n)}}\left(x_{1}, \ldots, x_{n}\right) \in \mathcal{E}_{n}
$$

The fundamental property of such an elementary polynomial is that for all Lie algebra generators

$$
\left(\sum_{l=1}^{n} \tilde{J}_{a}\left(x_{l}, \partial, \lambda_{(l)}\right)\right) R^{\lambda_{(1)}, \ldots, \lambda_{(n)}}\left(x_{1}, \ldots, x_{n}\right)=0
$$

For elements in $\mathcal{E}_{n}^{2}$, let us introduce the abbreviation

$$
R^{i}\left(x_{j}, x_{k}\right)=R^{\lambda_{(1)}, \ldots, \lambda_{(n)}}\left(x_{1}, \ldots, x_{n}\right) \text { if } \lambda_{(j)}=\Lambda^{i}, \lambda_{(k)}=\Lambda^{i^{+}}
$$




\subsubsection{Syzygies}

Even though the elements in $\mathcal{E}_{n}$ are linearly independent, there may exist algebraic relations between them. They appear when a given coupling may be represented non-uniquely in terms of elementary couplings, and are conventionally denoted syzygies. Correspondingly, we shall denote a non-trivial algebraic relation between elements of $\mathcal{E}_{n}$ a syzygy. Thus, in this wave function formalism syzygies appear as vanishing linear combinations of ordinary products of elementary polynomials.

\subsection{Solutions in Terms of Generalized Anharmonic Ratios}

The main result in this paper is a prescription for generating the complete and minimal set of solutions to the following general ansatz for the affine part of a 3-point function

$$
\begin{aligned}
& W_{3}^{a f f}\left(x_{1}, x_{2}, x_{3} ; \Lambda_{(1)}, \Lambda_{(2)}, \Lambda_{(3)}\right) \\
= & \prod_{i=1}^{r}\left(\left(R^{i}\left(x_{1}, x_{2}\right)\right)^{p_{i}(1,2)}\left(R^{i}\left(x_{2}, x_{3}\right)\right)^{p_{i}(2,3)}\left(R^{i}\left(x_{3}, x_{1}\right)\right)^{p_{i}(3,1)}\right) \\
\cdot & \prod_{\left(\lambda_{(1)}, \lambda_{(2)}, \lambda_{(3)}\right) \in \mathcal{L}_{3}^{3}}\left(R^{\lambda_{(1)}, \lambda_{(2)}, \lambda_{(3)}}\left(x_{1}, x_{2}, x_{3}\right)\right)^{p_{\lambda_{(1)}, \lambda_{(2)}, \lambda_{(3)}}}
\end{aligned}
$$

where the exponents $p_{i}(1,2), p_{i}(2,3), p_{i}(3,1)$ and $p_{\lambda_{(1)}, \lambda_{(2)}, \lambda_{(3)}}$ are functions of the weights $\Lambda_{(1)}, \Lambda_{(2)}$ and $\Lambda_{(3)}$. The complete solution is given by a linear combination of such monomials which, by construction, span the space of chiral blocks.

It is convenient to introduce the following conjugation operation. For any $r \times s$ matrix $M$ the conjugated $r \times s$ matrix $M^{+}$is defined by interchanging the $i$ 'th and $j^{\prime}$ th rows when $i^{+}=j$, that is when $\Lambda^{i^{+}}=\Lambda^{j}$ where $\left\{\Lambda^{i}\right\}_{i=1}^{r}$ is the set of fundamental weights. This is a straightforward generalization of the notion of the conjugate weight $\Lambda^{+}$to the weight $\Lambda$ since in this respect the weights are $r \times 1$ matrices.

Now, inserting the ansatz (31) in the affine Ward identities (21) leads to the following linear equation system in the exponents

$$
\left(\begin{array}{llll}
I & 0 & I^{+} & M_{1} \\
I^{+} & I & 0 & M_{2} \\
0 & I^{+} & I & M_{3}
\end{array}\right)\left(\begin{array}{l}
p_{1}(1,2) \\
\vdots \\
p_{r}(1,2) \\
p_{1}(2,3) \\
\vdots \\
p_{r}(2,3) \\
p_{1}(3,1) \\
\vdots \\
p_{r}(3,1) \\
p_{\lambda_{(1)}, \lambda_{(2)}, \lambda_{(3)}} \\
\vdots \\
p_{\lambda_{(1)}^{\prime}, \lambda_{(2)}^{\prime}, \lambda_{(3)}^{\prime}}
\end{array}\right)=\left(\begin{array}{l}
\Lambda_{(1)} \\
\Lambda_{(2)} \\
\Lambda_{(3)}
\end{array}\right)
$$


which may be recast into

$$
\left(\begin{array}{cccc}
I & 0 & -I & M_{2}^{+}-M_{3} \\
0 & I & -I & -M_{1}^{+}+M_{2} \\
0 & 0 & I+I^{+} & M_{1}-M_{2}^{+}+M_{3}
\end{array}\right)\left(\begin{array}{l}
p_{1}(1,2) \\
\vdots \\
p_{r}(1,2) \\
p_{1}(2,3) \\
\vdots \\
p_{r}(2,3) \\
p_{1}(3,1) \\
\vdots \\
p_{r}(3,1) \\
p_{\lambda_{(1)}, \lambda_{(2)}, \lambda_{(3)}} \\
\vdots \\
p_{\lambda_{(1)}^{\prime}, \lambda_{(2)}^{\prime}, \lambda_{(3)}^{\prime}}
\end{array}\right)=\left(\begin{array}{c}
\Lambda_{(2)}^{+}-\Lambda_{(3)} \\
-\Lambda_{(1)}^{+}+\Lambda_{(2)} \\
\Lambda_{(1)}-\Lambda_{(2)}^{+}+\Lambda_{(3)}
\end{array}\right)
$$

Note that the matrices $M_{1}, M_{2}$ and $M_{3}$ thus defined are $r \times\left(\operatorname{dim}\left(\mathcal{L}_{3}^{3}\right)\right)$ matrices where any ordering of the elements of $\mathcal{L}_{3}^{3}$ may be chosen. Since it is only certain representations in the $D_{r}$ series of classical simple Lie algebras and most representations in the $A_{r}$ and $E_{6}$ series that are not self-conjugate, the $B_{r}, C_{r}, E_{7}, E_{8}, F_{4}$ and $G_{2}$ series may be treated simultaneously in which cases the set of linear equations become

$$
\left(\begin{array}{cccc}
I & 0 & 0 & \frac{1}{2}\left(M_{1}+M_{2}-M_{3}\right) \\
0 & I & 0 & \frac{1}{2}\left(-M_{1}+M_{2}+M_{3}\right) \\
0 & 0 & I & \frac{1}{2}\left(M_{1}-M_{2}+M_{3}\right)
\end{array}\right)\left(\begin{array}{l}
p_{1}(1,2) \\
\vdots \\
p_{r}(1,2) \\
p_{1}(2,3) \\
\vdots \\
p_{r}(2,3) \\
p_{1}(3,1) \\
\vdots \\
p_{r}(3,1) \\
p_{\lambda_{(1)}, \lambda_{(2)}, \lambda_{(3)}} \\
\vdots \\
p_{\lambda_{(1)}^{\prime}, \lambda_{(2)}^{\prime}, \lambda_{(3)}^{\prime}}
\end{array}\right)=\frac{1}{2}\left(\begin{array}{c}
\Lambda_{(1)}+\Lambda_{(2)}-\Lambda_{(3)} \\
-\Lambda_{(1)}+\Lambda_{(2)}+\Lambda_{(3)} \\
\Lambda_{(1)}-\Lambda_{(2)}+\Lambda_{(3)}
\end{array}\right)
$$

The general solution to (32) may be parameterized by $a_{1}, \ldots, a_{n}$ where the number $n$ depends on the Lie algebra under consideration and is given by the dimension of $\mathcal{L}_{3}$ subtracted the rank of the matrix multiplying the exponent vector in (32). Correspondingly, there will be $n$ monomials $A_{1}, \ldots, A_{n}$ in the elementary polynomials satisfying

$$
\left(\sum_{l=1}^{3} V_{-\alpha_{i}}^{\beta}\left(x_{l}\right) \partial_{x_{l}^{\beta}}\right) A_{m}=0 \quad, \quad m=1, \ldots, n
$$

for all simple roots $\alpha_{i}$ (in fact for all roots). These will be denoted generalized anharmonic 
ratios. The terminology is inspired by the well known anharmonic ratio in $S L(2)$

$$
z=\frac{\left(z_{1}-z_{2}\right)\left(z_{3}-z_{4}\right)}{\left(z_{2}-z_{3}\right)\left(z_{4}-z_{1}\right)}
$$

satisfying a condition similar to (35), namely

$$
\begin{aligned}
\left(\sum_{l=1}^{4} V_{-\alpha}\left(z_{l}\right) \partial_{z_{l}}\right) z & =0 \\
V_{-\alpha}\left(z_{l}\right) & =-\left(z_{l}\right)^{2}
\end{aligned}
$$

In general, there are infinitely many solutions to the linear equation system (32). However, we are only interested in solutions pertaining to integrable representations where all exponents in (31) must be non-negative integers, leaving only a finite set of solutions. This number of solutions may in general exceed the tensor product coefficient for the coupling in question. The reduction is achieved by employing the syzygies. Indeed, following from those, the generalized anharmonic ratios satisfy certain algebraic relations which may be used in obtaining the final (reduced) set of independent 3-point functions, see Section 4. Thus, an interesting by-product is a general procedure for computing the tensor product coefficients, essentially by counting integer solutions to the inequalities ensuring non-negative exponents, and with the complete sets of elementary couplings and associated syzygies as the only basic (group theoretical) input.

Our construction is illustrated in Section 4 where we consider the generically non-selfconjugate cases $S L(3)$ and $S L(4)$ (based on the Lie algebras $A_{2}$ and $A_{3}$, respectively) and the self-conjugate case $S O(5)$ (based on the Lie algebra $B_{2}$ ). It should be stressed that the many explicit polynomials in Section 4 and Appendix A are only needed in order to obtain explicit expressions for the syzygies. These again are only needed in establishing the algebraic relations satisfied by the generalized anharmonic ratios 2 . Thus, it would be very convenient if one could device a general procedure for determining the latter relations or just the syzygies in our basis of elementary polynomials without employing explicit knowledge of the polynomials. This is currently under investigation. Nevertheless, in order to perform the reductions, only the form is needed of the algebraic relations satisfied by the generalized anharmonic ratios. We shall comment further on this when discussing the case of $S O(5)$ in Section 4.

\subsection{2-point Functions}

Here we review the result for the 2-point functions obtained in [1]. The 2-point function of the primary fields $\phi_{\Lambda_{(1)}}\left(z_{1}, x_{1}\right)$ and $\phi_{\Lambda_{(2)}}\left(z_{2}, x_{2}\right)$ in an affine current algebra is given by

$$
\begin{aligned}
& W_{2}\left(z_{1}, z_{2} ; x_{1}, x_{2} ; \Lambda_{(1)}, \Lambda_{(2)} ; k\right) \\
= & C_{\Lambda_{(1)} \Lambda_{(2)}}(k) \frac{\delta_{\Delta\left(\phi_{\Lambda_{(1)}}\right), \Delta\left(\phi_{\Lambda_{(2)}}\right)}}{\left(z_{1}-z_{2}\right)^{\Delta\left(\phi_{\Lambda_{(1)}}\right)+\Delta\left(\phi_{\Lambda_{(2)}}\right)}} \prod_{i=1}^{r}\left(R^{i}\left(x_{1}, x_{2}\right)\right)^{p_{i}\left(\Lambda_{(1)}, \Lambda_{(2)}\right)}
\end{aligned}
$$

\footnotetext{
${ }^{2}$ The algebraic relations satisfied by the anharmonic ratios depend on the normalizations of the elementary polynomials. However, qua (25) these are fixed by the choice of basis for the vectors in the highest weight module.
} 
where

$$
p_{i}\left(\Lambda_{(1)}, \Lambda_{(2)}\right)=\alpha_{i}^{\vee} \cdot \Lambda_{(1)}=\alpha_{i^{+}}^{\vee} \cdot \Lambda_{(2)}
$$

This result is valid for all representations, integrable or non-integrable. It follows immediately that the conjugate weight $\Lambda^{+}$to an arbitrary weight $\Lambda$, integrable or non-integrable, is given by (23).

\section{Explicit Results for 3-point Functions}

In this section we shall illustrate our procedure by considering the 3 cases $S L(3), S O(5)$ and $S L(4)$. Many technical details are referred to Appendix A. The simplest case of $S L(2)$ is trivial since the loop projective invariance fixes completely the $x$ dependence, to a form analogous to the $z$ dependence (19). This is a well known result. For higher groups and to the best of our knowledge, the work [5] by Rühl is the only one discussing general aspects of 3-point functions using triangular coordinates. However, his construction is designed explicitly to cover $S L(N)$ only and differs considerably from ours, see below.

\subsection{Case of $S L(3)$}

The Lie algebra underlying affine $S L(3)$ current algebra is $A_{2}$ which has rank $r=2$ and one non-simple root, the highest root $\theta=\alpha_{1}+\alpha_{2}$. According to the general discussion above, we need the explicit differential operator realization of the two lowering Chevalley generators which are easily found to be [6]

$$
\begin{aligned}
& \tilde{F}_{\alpha_{1}}(x, \partial, \Lambda)=-\left(x^{1}\right)^{2} \partial_{1}+\left(\frac{1}{2} x^{1} x^{2}-x^{\theta}\right) \partial_{2}-\frac{1}{2} x^{1}\left(\frac{1}{2} x^{1} x^{2}+x^{\theta}\right) \partial_{\theta}+x^{1} \Lambda_{1} \\
& \tilde{F}_{\alpha_{2}}(x, \partial, \Lambda)=\left(\frac{1}{2} x^{1} x^{2}+x^{\theta}\right) \partial_{1}-\left(x^{2}\right)^{2} \partial_{2}+\frac{1}{2} x^{2}\left(\frac{1}{2} x^{1} x^{2}-x^{\theta}\right) \partial_{\theta}+x^{2} \Lambda_{2}
\end{aligned}
$$

Here we have introduced the abbreviations $\partial_{1}=\partial_{\alpha_{1}}$ etc. The elementary couplings are

$$
\begin{aligned}
\mathcal{L}_{3}^{2}= & \left\{\left(\Lambda^{1}, \Lambda^{2}, 0\right),\left(0, \Lambda^{1}, \Lambda^{2}\right),\left(\Lambda^{2}, 0, \Lambda^{1}\right),\right. \\
& \left.\left(\Lambda^{2}, \Lambda^{1}, 0\right),\left(0, \Lambda^{2}, \Lambda^{1}\right),\left(\Lambda^{1}, 0, \Lambda^{2}\right)\right\} \\
\mathcal{L}_{3}^{3}= & \left\{\left(\Lambda^{1}, \Lambda^{1}, \Lambda^{1}\right),\left(\Lambda^{2}, \Lambda^{2}, \Lambda^{2}\right)\right\}
\end{aligned}
$$

It is straightforward to work out explicit representations of the singlets. As an illustration,

$$
\begin{aligned}
\left|\operatorname{singlet}\left(\Lambda^{1}, \Lambda^{1}, \Lambda^{1}\right)\right\rangle & =|1,0\rangle \otimes|-1,1\rangle \otimes|0,-1\rangle-|1,0\rangle \otimes|0,-1\rangle \otimes|-1,1\rangle \\
& +|-1,1\rangle \otimes|0,-1\rangle \otimes|1,0\rangle-|-1,1\rangle \otimes|1,0\rangle \otimes|0,-1\rangle \\
& +|0,-1\rangle \otimes|1,0\rangle \otimes|-1,1\rangle-|0,-1\rangle \otimes|-1,1\rangle \otimes|1,0\rangle
\end{aligned}
$$

is the singlet in the elementary coupling $\left(\Lambda^{1}, \Lambda^{1}, \Lambda^{1}\right) \in \mathcal{L}_{3}^{3}$. Here the vector notation refers to the elements in the fundamental module with highest weight $\Lambda^{1}$ where a vector of weight $\Lambda$ is denoted by its Dynkin labels

$$
|\Lambda\rangle=\left|\Lambda_{1}, \ldots, \Lambda_{r}\right\rangle
$$


The basis vectors in the two fundamental representation spaces are

$$
\begin{array}{rlrl}
|1,0\rangle & |0,1\rangle & \\
|-1,1\rangle & =f_{1}|1,0\rangle & |1,-1\rangle & =f_{2}|0,1\rangle \\
|0,-1\rangle & =f_{2}|-1,1\rangle & |-1,0\rangle & =f_{1}|1,-1\rangle
\end{array}
$$

After having introduced the shorthand notation

$$
x_{i}^{ \pm}=\frac{1}{2} x_{i}^{1} x_{i}^{2} \pm x_{i}^{\theta}
$$

the elementary polynomials may be written

$$
\begin{aligned}
R^{1}\left(x_{1}, x_{2}\right) & =x_{1}^{+}+x_{2}^{-}-x_{1}^{1} x_{2}^{2} \\
R^{1}\left(x_{2}, x_{3}\right) & =x_{2}^{+}+x_{3}^{-}-x_{2}^{1} x_{3}^{2} \\
R^{1}\left(x_{3}, x_{1}\right) & =x_{3}^{+}+x_{1}^{-}-x_{3}^{1} x_{1}^{2} \\
R^{2}\left(x_{1}, x_{2}\right) & =x_{1}^{-}+x_{2}^{+}-x_{1}^{2} x_{2}^{1} \\
R^{2}\left(x_{2}, x_{3}\right) & =x_{2}^{-}+x_{3}^{+}-x_{2}^{2} x_{3}^{1} \\
R^{2}\left(x_{3}, x_{1}\right) & =x_{3}^{-}+x_{1}^{+}-x_{3}^{2} x_{1}^{1} \\
R^{1,1,1}\left(x_{1}, x_{2}, x_{3}\right) & =x_{2}^{1} x_{3}^{+}-x_{2}^{+} x_{3}^{1}+x_{1}^{1}\left(x_{2}^{+}-x_{3}^{+}\right)+x_{1}^{+}\left(x_{3}^{1}-x_{2}^{1}\right) \\
R^{2,2,2}\left(x_{1}, x_{2}, x_{3}\right) & =x_{2}^{2} x_{3}^{-}-x_{2}^{-} x_{3}^{2}+x_{1}^{2}\left(x_{2}^{-}-x_{3}^{-}\right)+x_{1}^{-}\left(x_{3}^{2}-x_{2}^{2}\right)
\end{aligned}
$$

In this basis the general ansatz for the $x$ dependent part of the 3-point function becomes

$$
\begin{aligned}
& W_{3}^{a f f}\left(x_{1}, x_{2}, x_{3} ; \Lambda_{(1)}, \Lambda_{(2)}, \Lambda_{(3)}\right) \\
= & \prod_{i=1,2}\left(\left(R^{i}\left(x_{1}, x_{2}\right)\right)^{p_{i}(1,2)}\left(R^{i}\left(x_{2}, x_{3}\right)\right)^{p_{i}(2,3)}\left(R^{i}\left(x_{3}, x_{1}\right)\right)^{p_{i}(3,1)}\right) \\
\cdot & \left(R^{1,1,1}\left(x_{1}, x_{2}, x_{3}\right)\right)^{p_{1,1,1}}\left(R^{2,2,2}\left(x_{1}, x_{2}, x_{3}\right)\right)^{p_{2,2,2}}
\end{aligned}
$$

It is easily verified that with the ordering $p_{1,1,1}, p_{2,2,2}$ the 3 matrices $M_{1}, M_{2}$ and $M_{3}$ in (32) are all identical to the $2 \times 2$ unit matrix while the conjugate of any $2 \times s$ matrix is given by interchanging the two rows $\left(\right.$ since $\left.\left(\Lambda^{1}\right)^{+}=\Lambda^{2}\right)$. Thus, the exponents are given by

$$
\begin{aligned}
p_{1}(1,2) & =\alpha_{1}^{\vee} \cdot \Lambda_{(1)}-\frac{1}{3}\left(\alpha_{1}^{\vee}-\alpha_{2}^{\vee}\right) \cdot\left(\Lambda_{(1)}+\Lambda_{(2)}+\Lambda_{(3)}\right)-\left(a_{1}+a_{2}\right) \\
p_{2}(1,2) & =\alpha_{1}^{\vee} \cdot \Lambda_{(2)}-\alpha_{2}^{\vee} \cdot \Lambda_{(3)}-\frac{1}{3}\left(\alpha_{1}^{\vee}-\alpha_{2}^{\vee}\right) \cdot\left(\Lambda_{(1)}+\Lambda_{(2)}+\Lambda_{(3)}\right)+a_{1} \\
p_{1}(2,3) & =\alpha_{2}^{\vee} \cdot \Lambda_{(3)}-\left(a_{1}+a_{2}\right) \\
p_{2}(2,3) & =-\alpha_{1}^{\vee} \cdot \Lambda_{(1)}+\alpha_{2}^{\vee} \cdot \Lambda_{(2)}+\frac{1}{3}\left(\alpha_{1}^{\vee}-\alpha_{2}^{\vee}\right) \cdot\left(\Lambda_{(1)}+\Lambda_{(2)}+\Lambda_{(3)}\right)+a_{1} \\
p_{1}(3,1) & =\frac{1}{3} \alpha_{1}^{\vee} \cdot\left(\Lambda_{(1)}-2 \Lambda_{(2)}+\Lambda_{(3)}\right)+\frac{1}{3} \alpha_{2}^{\vee} \cdot\left(2 \Lambda_{(1)}-\Lambda_{(2)}+2 \Lambda_{(3)}\right)-\left(a_{1}+a_{2}\right) \\
p_{2}(3,1) & =a_{1} \\
p_{1,1,1} & =\frac{1}{3}\left(\alpha_{1}^{\vee}-\alpha_{2}^{\vee}\right) \cdot\left(\Lambda_{(1)}+\Lambda_{(2)}+\Lambda_{(3)}\right)+a_{2} \\
p_{2,2,2} & =a_{2}
\end{aligned}
$$


parameterized by $a_{1}$ and $a_{2}$, while the generalized anharmonic ratios become

$$
\begin{aligned}
& A_{1}=\frac{R^{2}\left(x_{1}, x_{2}\right) R^{2}\left(x_{2}, x_{3}\right) R^{2}\left(x_{3}, x_{1}\right)}{R^{1}\left(x_{1}, x_{2}\right) R^{1}\left(x_{2}, x_{3}\right) R^{1}\left(x_{3}, x_{1}\right)} \\
& A_{2}=\frac{R^{1,1,1}\left(x_{1}, x_{2}, x_{3}\right) R^{2,2,2}\left(x_{1}, x_{2}, x_{3}\right)}{R^{1}\left(x_{1}, x_{2}\right) R^{1}\left(x_{2}, x_{3}\right) R^{1}\left(x_{3}, x_{1}\right)}
\end{aligned}
$$

The single syzygy appears for the coupling $\left(\Lambda^{1}+\Lambda^{2}, \Lambda^{1}+\Lambda^{2}, \Lambda^{1}+\Lambda^{2}\right)$ and is worked out to be

$$
\begin{aligned}
R^{1,1,1}\left(x_{1}, x_{2}, x_{3}\right) R^{2,2,2}\left(x_{1}, x_{2}, x_{3}\right) & =R^{1}\left(x_{1}, x_{2}\right) R^{1}\left(x_{2}, x_{3}\right) R^{1}\left(x_{3}, x_{1}\right) \\
& +R^{2}\left(x_{1}, x_{2}\right) R^{2}\left(x_{2}, x_{3}\right) R^{2}\left(x_{3}, x_{1}\right)
\end{aligned}
$$

This means that the generalized anharmonic ratios are related as

$$
A_{2}=1+A_{1}
$$

It is recalled that $\Lambda^{1}+\Lambda^{2}=\theta$ is the adjoint representation.

Let us illustrate the procedure for obtaining the space of chiral blocks by considering the coupling above where

$$
\Lambda_{(1)}=\Lambda_{(2)}=\Lambda_{(3)}=\Lambda^{1}+\Lambda^{2}
$$

The condition that all powers (48) should be non-negative integers reduces to

$$
a_{1}, a_{2}, 1-a_{1}-a_{2} \geq 0
$$

with the obvious solution

$$
\left(a_{1}, a_{2}\right) \in\{(0,0),(0,1),(1,0)\}
$$

so the space of chiral blocks is generated by the functions

$$
\left\{1, A_{1}, A_{2}\right\} \rightarrow\left\{1, A_{1}\right\}
$$

The reduction is due to the syzygy. In conclusion, the space of chiral blocks is 2 dimensional (in accordance with the well known result that the corresponding tensor product coefficient is 2) and is spanned by

$$
\begin{aligned}
& W_{3}^{a f f, 1}=R^{1}\left(x_{1}, x_{2}\right) R^{1}\left(x_{2}, x_{3}\right) R^{1}\left(x_{3}, x_{1}\right) \\
& W_{3}^{a f f, 2}=R^{2}\left(x_{1}, x_{2}\right) R^{2}\left(x_{2}, x_{3}\right) R^{2}\left(x_{3}, x_{1}\right)
\end{aligned}
$$

\subsection{Case of $S O(5)$}

Here we shall consider the case $S O(5)$ where the affine current algebra is based on the simple Lie algebra $B_{2}$. This Lie algebra has rank $r=2$ and two non-simple positive roots denoted

$$
\alpha_{11}=\alpha_{1}+\alpha_{2} \quad, \quad \theta=\alpha_{1}+2 \alpha_{2}
$$


A discussion of $S O(5)$ may be found in [11] and the result presented there relevant for our purpose is the following explicit expression for the differential operator realization of the two lowering Chevalley operators

$$
\begin{aligned}
\tilde{F}_{\alpha_{1}}(x, \partial, \Lambda) & =-x^{1} x^{1} \partial_{1}+\left(\frac{1}{2} x^{1} x^{2}-x^{11}\right) \partial_{2}-\frac{1}{2} x^{1}\left(\frac{1}{2} x^{1} x^{2}+x^{11}\right) \partial_{11} \\
& +\frac{1}{3} x^{1} x^{2} x^{11} \partial_{\theta}+x^{1} \Lambda_{1} \\
\tilde{F}_{\alpha_{2}}(x, \partial, \Lambda) & =2\left(\frac{1}{2} x^{1} x^{2}+x^{11}\right) \partial_{1}-x^{2} x^{2} \partial_{2}+\left(\frac{1}{3} x^{1} x^{2} x^{2}-x^{\theta}\right) \partial_{11} \\
& -x^{2}\left(\frac{1}{3} x^{2} x^{11}+x^{\theta}\right) \partial_{\theta}+x^{2} \Lambda_{2}
\end{aligned}
$$

Here we have introduced the abbreviations $x^{11}=x^{\alpha_{11}}, \partial_{1}=\partial_{\alpha_{1}}$ etc. The elementary couplings are

$$
\begin{aligned}
\mathcal{L}_{3}^{2}= & \left\{\left(\Lambda^{1}, \Lambda^{1}, 0\right),\left(\Lambda^{1}, 0, \Lambda^{1}\right),\left(0, \Lambda^{1}, \Lambda^{1}\right),\right. \\
& \left.\left(\Lambda^{2}, \Lambda^{2}, 0\right),\left(0, \Lambda^{2}, \Lambda^{2}\right),\left(\Lambda^{2}, 0, \Lambda^{2}\right)\right\} \\
\mathcal{L}_{3}^{3}= & \left\{\left(\Lambda^{1}, \Lambda^{2}, \Lambda^{2}\right),\left(\Lambda^{2}, \Lambda^{1}, \Lambda^{2}\right),\left(\Lambda^{2}, \Lambda^{2}, \Lambda^{1}\right),\right. \\
& \left.\left(2 \Lambda^{2}, \Lambda^{1}, \Lambda^{1}\right),\left(\Lambda^{1}, 2 \Lambda^{2}, \Lambda^{1}\right),\left(\Lambda^{1}, \Lambda^{1}, 2 \Lambda^{2}\right)\right\}
\end{aligned}
$$

Thus, the general ansatz for the affine part of the 3-point function becomes

$$
\begin{aligned}
& W_{3}^{a f f}\left(x_{1}, x_{2}, x_{3} ; \Lambda_{(1)}, \Lambda_{(2)}, \Lambda_{(3)}\right) \\
= & \prod_{i=1,2}\left(\left(R^{i}\left(x_{1}, x_{2}\right)\right)^{p_{i}(1,2)}\left(R^{i}\left(x_{2}, x_{3}\right)\right)^{p_{i}(2,3)}\left(R^{i}\left(x_{3}, x_{1}\right)\right)^{p_{i}(3,1)}\right) \\
\cdot & \left(R^{1,2,2}\left(x_{1}, x_{2}, x_{3}\right)\right)^{p_{1,2,2}}\left(R^{2,1,2}\left(x_{1}, x_{2}, x_{3}\right)\right)^{p_{2,1,2}}\left(R^{2,2,1}\left(x_{1}, x_{2}, x_{3}\right)\right)^{p_{2,2,1}} \\
\cdot & \left(R^{4,1,1}\left(x_{1}, x_{2}, x_{3}\right)\right)^{p_{4,1,1}}\left(R^{1,4,1}\left(x_{1}, x_{2}, x_{3}\right)\right)^{p_{1,4,1}}\left(R^{1,1,4}\left(x_{1}, x_{2}, x_{3}\right)\right)^{p_{1,1,4}}
\end{aligned}
$$

where $p_{1,2,2}, p_{2,1,2}, p_{2,2,1}, p_{4,1,1}, p_{1,4,1}, p_{1,1,4}$ is a natural ordering of the exponents. Here we have introduced the abbreviations $(1,2,2),(4,1,1)$ etc to denote the elementary couplings $\left(\Lambda^{1}, \Lambda^{2}, \Lambda^{2}\right),\left(2 \Lambda^{2}, \Lambda^{1}, \Lambda^{1}\right)$ etc. Explicit expressions for the elementary polynomials are provided in Appendix A. With this ansatz we find that the three matrices $M_{1}, M_{2}$ and $M_{3}$ in (34) are

$$
\begin{aligned}
& M_{1}=\left(\begin{array}{llllll}
1 & 0 & 0 & 0 & 1 & 1 \\
0 & 1 & 1 & 2 & 0 & 0
\end{array}\right) \\
& M_{2}=\left(\begin{array}{llllll}
0 & 1 & 0 & 1 & 0 & 1 \\
1 & 0 & 1 & 0 & 2 & 0
\end{array}\right) \\
& M_{3}=\left(\begin{array}{llllll}
0 & 0 & 1 & 1 & 1 & 0 \\
1 & 1 & 0 & 0 & 0 & 2
\end{array}\right)
\end{aligned}
$$

The solution to (34) in terms of the exponents may be written

$$
p_{1}(1,2)=\frac{1}{2} \alpha_{1}^{\vee} \cdot\left(\Lambda_{(1)}+\Lambda_{(2)}-\Lambda_{(3)}\right)-\frac{1}{2}\left(a_{1}+a_{2}-a_{3}+2 a_{6}\right)
$$




$$
\begin{aligned}
p_{2}(1,2) & =\frac{1}{2} \alpha_{2}^{\vee} \cdot\left(\Lambda_{(1)}+\Lambda_{(2)}-\Lambda_{(3)}\right)-\left(a_{3}+a_{4}+a_{5}-a_{6}\right) \\
p_{1}(2,3) & =\frac{1}{2} \alpha_{1}^{\vee} \cdot\left(-\Lambda_{(1)}+\Lambda_{(2)}+\Lambda_{(3)}\right)-\frac{1}{2}\left(-a_{1}+a_{2}+a_{3}+2 a_{4}\right) \\
p_{2}(2,3) & =\frac{1}{2} \alpha_{2}^{\vee} \cdot\left(-\Lambda_{(1)}+\Lambda_{(2)}+\Lambda_{(3)}\right)-\left(a_{1}-a_{4}+a_{5}+a_{6}\right) \\
p_{1}(3,1) & =\frac{1}{2} \alpha_{1}^{\vee} \cdot\left(\Lambda_{(1)}-\Lambda_{(2)}+\Lambda_{(3)}\right)-\frac{1}{2}\left(a_{1}-a_{2}+a_{3}+2 a_{5}\right) \\
p_{2}(3,1) & =\frac{1}{2} \alpha_{2}^{\vee} \cdot\left(\Lambda_{(1)}-\Lambda_{(2)}+\Lambda_{(3)}\right)-\left(a_{2}+a_{4}-a_{5}+a_{6}\right) \\
p_{1,2,2} & =a_{1} \quad, \quad p_{2,1,2}=a_{2} \quad, \quad p_{2,2,1}=a_{3} \\
p_{4,1,1} & =a_{4} \quad, \quad p_{1,4,1}=a_{5} \quad, \quad p_{1,1,4}=a_{6}
\end{aligned}
$$

The generalized anharmonic ratios are readily seen to be

$$
\begin{aligned}
& A_{1}=\frac{\left(R^{1}\left(x_{2}, x_{3}\right)\right)^{\frac{1}{2}} R^{1,2,2}\left(x_{1}, x_{2}, x_{3}\right)}{\left(R^{1}\left(x_{1}, x_{2}\right)\right)^{\frac{1}{2}} R^{2}\left(x_{2}, x_{3}\right)\left(R^{1}\left(x_{3}, x_{1}\right)\right)^{\frac{1}{2}}} \\
& A_{2}=\frac{\left(R^{1}\left(x_{3}, x_{1}\right)\right)^{\frac{1}{2}} R^{2,1,2}\left(x_{1}, x_{2}, x_{3}\right)}{\left(R^{1}\left(x_{1}, x_{2}\right)\right)^{\frac{1}{2}}\left(R^{1}\left(x_{2}, x_{3}\right)\right)^{\frac{1}{2}} R^{2}\left(x_{3}, x_{1}\right)} \\
& A_{3}=\frac{\left(R^{1}\left(x_{1}, x_{2}\right)\right)^{\frac{1}{2}} R^{2,2,1}\left(x_{1}, x_{2}, x_{3}\right)}{R^{2}\left(x_{1}, x_{2}\right)\left(R^{1}\left(x_{2}, x_{3}\right)\right)^{\frac{1}{2}}\left(R^{1}\left(x_{3}, x_{1}\right)\right)^{\frac{1}{2}}} \\
& A_{4}=\frac{R^{2}\left(x_{2}, x_{3}\right) R^{4,1,1}\left(x_{1}, x_{2}, x_{3}\right)}{R^{2}\left(x_{1}, x_{2}\right) R^{1}\left(x_{2}, x_{3}\right) R^{2}\left(x_{3}, x_{1}\right)} \\
& A_{5}=\frac{R^{2}\left(x_{3}, x_{1}\right) R^{1,4,1}\left(x_{1}, x_{2}, x_{3}\right)}{R^{2}\left(x_{1}, x_{2}\right) R^{2}\left(x_{2}, x_{3}\right) R^{1}\left(x_{3}, x_{1}\right)} \\
& A_{6}=\frac{R^{2}\left(x_{1}, x_{2}\right) R^{1,1,4}\left(x_{1}, x_{2}, x_{3}\right)}{R^{1}\left(x_{1}, x_{2}\right) R^{2}\left(x_{2}, x_{3}\right) R^{2}\left(x_{3}, x_{1}\right)}
\end{aligned}
$$

Due to the syzygies listed in Appendix A, the generalized anharmonic ratios satisfy the following set of non-trivial algebraic relations

$$
\begin{aligned}
& 0=A_{4}+t_{1} A_{2} A_{3}+u_{1} \\
& 0=A_{5}+t_{2} A_{1} A_{3}+u_{2} \\
& 0=A_{6}+t_{3} A_{1} A_{2}+u_{3} \\
& 0=A_{1} A_{4}+t_{4} A_{2}+u_{4} A_{3} \\
& 0=A_{2} A_{5}+t_{5} A_{1}+u_{5} A_{3} \\
& 0=A_{3} A_{6}+t_{6} A_{1}+u_{6} A_{2} \\
& 0=A_{4} A_{5}+t_{7}\left(A_{3}\right)^{2}+u_{7} \\
& 0=A_{4} A_{6}+t_{8}\left(A_{2}\right)^{2}+u_{8} \\
& 0=A_{5} A_{6}+t_{9}\left(A_{1}\right)^{2}+u_{9}
\end{aligned}
$$

The parameters $\left(u_{\ell}, t_{\ell}\right), \ell=1, \ldots, 9$ are given in (91) in Appendix A.

Let us illustrate the procedure by considering the case

$$
\Lambda_{(1)}=(0,2) \quad, \quad \Lambda_{(2)}=(1,1) \quad, \quad \Lambda_{(3)}=(1,1)
$$


One may show that the corresponding tensor product coefficient is 2 . Thus, we expect to find a 2 dimensional solution space for the chiral blocks. Now, the condition that all exponents should be non-negative integers reduces to

$$
\begin{aligned}
0 & \leq a_{1}, \ldots, a_{6} \\
0 & \leq \frac{1}{2}\left(-a_{1}-a_{2}+a_{3}-2 a_{6}\right) \\
0 & \leq 1-a_{3}-a_{4}-a_{5}+a_{6} \\
0 & \leq 1+\frac{1}{2}\left(a_{1}-a_{2}-a_{3}-2 a_{4}\right) \\
0 & \leq-a_{1}+a_{4}-a_{5}-a_{6} \\
0 & \leq \frac{1}{2}\left(-a_{1}+a_{2}-a_{3}-2 a_{5}\right) \\
0 & \leq 1-a_{2}-a_{4}+a_{5}-a_{6}
\end{aligned}
$$

with solution

$$
\left(a_{1}, a_{2}, a_{3}, a_{4}, a_{5}, a_{6}\right) \in\{(0, \ldots, 0),(0,1,1,0,0,0),(0,0,0,1,0,0)\}
$$

This means that the space of chiral blocks is generated by

$$
\left\{1, A_{2} A_{3}, A_{4}\right\}
$$

However, due to (64) this set may be reduced to

$$
\left\{1, A_{4}\right\}
$$

which agrees with the tensor product coefficient being 2 . In conclusion, the space of chiral blocks is 2 dimensional and is spanned by

$$
\begin{aligned}
& W_{3}^{a f f, 1}=R^{2}\left(x_{1}, x_{2}\right) R^{1}\left(x_{2}, x_{3}\right) R^{2}\left(x_{3}, x_{1}\right) \\
& W_{3}^{a f f, 2}=R^{2}\left(x_{2}, x_{3}\right) R^{4,1,1}\left(x_{1}, x_{2}, x_{3}\right)
\end{aligned}
$$

A seemingly less trivial, yet simpler example is provided by the case

$$
\Lambda_{(1)}=(2,0) \quad, \quad \Lambda_{(2)}=(0,2) \quad, \quad \Lambda_{(3)}=(2,2)
$$

where we find that $a_{l}=0, l=1, \ldots, 6$ in order for the exponents to be non-negative integers. Thus, the single chiral block is

$$
W_{3}^{a f f}=\left(R^{2}\left(x_{2}, x_{3}\right) R^{1}\left(x_{3}, x_{1}\right)\right)^{2}
$$

Note that the reduction in (69) may be inferred even without knowing explicitly the parameters $\left(t_{\ell}, u_{\ell}\right)$ appearing in (64) (more generally $\left(s_{\ell}, t_{\ell}, u_{\ell}\right)$ etc, see Appendix A). All that is required is the knowledge whether or not they are non-vanishing since the vanishing of a parameter alters the form of the syzygy. This general feature of our formalism simplifies considerably the task of eliminating the redundancies due to the existence of syzygies. Nevertheless, in Appendix A we have provided the relevant parameters in the cases $S O(5)$ and $S L(4)$. 


\subsection{Case of $S L(4)$}

The Lie algebra underlying affine $S L(4)$ current algebra is $A_{3}$ which has rank $r=3$ and three non-simple roots denoted

$$
\alpha_{12}=\alpha_{1}+\alpha_{2} \quad, \quad \alpha_{23}=\alpha_{2}+\alpha_{3} \quad, \quad \theta=\alpha_{1}+\alpha_{2}+\alpha_{3}
$$

The relevant differential operators are worked out to be

$$
\begin{aligned}
\tilde{F}_{\alpha_{1}}(x, \partial, \Lambda) & =-\left(x^{1}\right)^{2} \partial_{1}-\frac{1}{2} x^{1}\left(x^{12}+\frac{1}{2} x^{1} x^{2}\right) \partial_{12}+\left(\frac{1}{2} x^{1} x^{2}-x^{12}\right) \partial_{2} \\
& +\left(-x^{\theta}+\frac{1}{2} x^{1} x^{23}+\frac{1}{12} x^{1} x^{2} x^{3}\right) \partial_{23}-\frac{1}{2} x^{1}\left(x^{\theta}+\frac{1}{2} x^{1} x^{23}+\frac{1}{6} x^{12} x^{3}\right) \partial_{\theta} \\
& +x^{1} \Lambda_{1} \\
\tilde{F}_{\alpha_{2}}(x, \partial, \Lambda) & =\left(x^{12}+\frac{1}{2} x^{1} x^{2}\right) \partial_{1}+\frac{1}{2} x^{2}\left(\frac{1}{2} x^{1} x^{2}-x^{12}\right) \partial_{12}-\left(x^{2}\right)^{2} \partial_{2} \\
& -\frac{1}{2} x^{2}\left(\frac{1}{2} x^{2} x^{3}+x^{23}\right) \partial_{23}+\left(\frac{1}{2} x^{2} x^{3}-x^{23}\right) \partial_{3}+\frac{1}{6} x^{2}\left(x^{1} x^{23}-x^{12} x^{3}\right) \partial_{\theta} \\
& +x^{2} \Lambda_{2} \\
& =\left(x^{\theta}+\frac{1}{2} x^{12} x^{3}-\frac{1}{12} x^{1} x^{2} x^{3}\right) \partial_{12}+\left(\frac{1}{2} x^{2} x^{3}+x^{23}\right) \partial_{2}-\left(x^{3}\right)^{2} \partial_{3} \\
\tilde{F}_{\alpha_{3}}(x, \partial, \Lambda) & \frac{1}{2} x^{3}\left(\frac{1}{2} x^{2} x^{3}-x^{23}\right) \partial_{23}+\frac{1}{2} x^{3}\left(-x^{\theta}+\frac{1}{2} x^{12} x^{3}+\frac{1}{6} x^{1} x^{12}\right) \partial_{\theta} \\
& +x^{3} \Lambda_{3}
\end{aligned}
$$

The elementary couplings are

$$
\begin{aligned}
\mathcal{L}_{3}^{2}= & \left(\Lambda^{1}, \Lambda^{3}, 0\right),\left(0, \Lambda^{1}, \Lambda^{3}\right),\left(\Lambda^{3}, 0, \Lambda^{1}\right), \\
& \left(\Lambda^{2}, \Lambda^{2}, 0\right),\left(0, \Lambda^{2}, \Lambda^{2}\right),\left(\Lambda^{2}, 0, \Lambda^{2}\right), \\
& \left.\left(\Lambda^{3}, \Lambda^{1}, 0\right),\left(0, \Lambda^{3}, \Lambda^{1}\right),\left(\Lambda^{1}, 0, \Lambda^{3}\right)\right\} \\
\mathcal{L}_{3}^{3}= & \left(\Lambda^{1}, \Lambda^{1}, \Lambda^{2}\right),\left(\Lambda^{1}, \Lambda^{2}, \Lambda^{1}\right),\left(\Lambda^{2}, \Lambda^{1}, \Lambda^{1}\right), \\
& \left(\Lambda^{2}, \Lambda^{3}, \Lambda^{3}\right),\left(\Lambda^{3}, \Lambda^{2}, \Lambda^{3}\right),\left(\Lambda^{3}, \Lambda^{3}, \Lambda^{2}\right), \\
& \left.\left(\Lambda^{1}+\Lambda^{3}, \Lambda^{2}, \Lambda^{2}\right),\left(\Lambda^{2}, \Lambda^{1}+\Lambda^{3}, \Lambda^{2}\right),\left(\Lambda^{2}, \Lambda^{2}, \Lambda^{1}+\Lambda^{3}\right)\right\}
\end{aligned}
$$

Thus, the general ansatz for the affine part of the 3-point function becomes

$$
\begin{aligned}
& W_{3}^{a f f}\left(x_{1}, x_{2}, x_{3} ; \Lambda_{(1)}, \Lambda_{(2)}, \Lambda_{(3)}\right) \\
= & \prod_{i=1}^{3}\left(\left(R^{i}\left(x_{1}, x_{2}\right)\right)^{p_{i}(1,2)}\left(R^{i}\left(x_{2}, x_{3}\right)\right)^{p_{i}(2,3)}\left(R^{i}\left(x_{3}, x_{1}\right)\right)^{p_{i}(3,1)}\right) \\
\cdot & \left(R^{1,1,2}\left(x_{1}, x_{2}, x_{3}\right)\right)^{p_{1,1,2}}\left(R^{1,2,1}\left(x_{1}, x_{2}, x_{3}\right)\right)^{p_{1,2,1}}\left(R^{2,1,1}\left(x_{1}, x_{2}, x_{3}\right)\right)^{p_{2,1,1}} \\
\cdot & \left(R^{2,3,3}\left(x_{1}, x_{2}, x_{3}\right)\right)^{p_{2,3,3}}\left(R^{3,2,3}\left(x_{1}, x_{2}, x_{3}\right)\right)^{p_{3,2,3}}\left(R^{3,3,2}\left(x_{1}, x_{2}, x_{3}\right)\right)^{p_{3,3,2}} \\
\cdot & \left(R^{4,2,2}\left(x_{1}, x_{2}, x_{3}\right)\right)^{p_{4,2,2}}\left(R^{2,4,2}\left(x_{1}, x_{2}, x_{3}\right)\right)^{p_{2,4,2}}\left(R^{2,2,4}\left(x_{1}, x_{2}, x_{3}\right)\right)^{p_{2,2,4}}
\end{aligned}
$$

where $p_{1,1,2}, p_{1,2,1}, p_{2,1,1}, p_{2,3,3}, p_{3,2,3}, p_{3,3,2}, p_{4,2,2}, p_{2,4,2}, p_{2,2,4}$ is a natural ordering of the exponents. Here we have introduced the abbreviations $(1,1,2),(2,4,2)$ etc to denote the 
elementary couplings $\left(\Lambda^{1}, \Lambda^{1}, \Lambda^{2}\right),\left(\Lambda^{2}, \Lambda^{1}+\Lambda^{3}, \Lambda^{2}\right)$ etc. Explicit expressions for the elementary polynomials are provided in Appendix A. We find the following solution to the linear equation system (32)

$$
\begin{aligned}
& p_{1}(1,2)=\frac{1}{2} \alpha_{1}^{\vee} \cdot\left(\Lambda_{(1)}-\Lambda_{(2)}-\Lambda_{(3)}\right)+\frac{1}{2} \alpha_{3}^{\vee} \cdot\left(\Lambda_{(1)}+\Lambda_{(2)}+\Lambda_{(3)}\right) \\
& -\left(a_{1}-a_{3}+a_{4}+a_{5}+a_{6}+a_{7}\right) \\
& p_{2}(1,2)=\frac{1}{2} \alpha_{2}^{\vee} \cdot\left(\Lambda_{(1)}+\Lambda_{(2)}-\Lambda_{(3)}\right)+\frac{1}{4}\left(\alpha_{1}^{\vee}-\alpha_{3}^{\vee}\right) \cdot\left(\Lambda_{(1)}+\Lambda_{(2)}+\Lambda_{(3)}\right) \\
& -\left(a_{2}+a_{3}-a_{6}+a_{9}\right) \\
& p_{3}(1,2)=\frac{1}{2} \alpha_{1}^{\vee} \cdot\left(-\Lambda_{(1)}+\Lambda_{(2)}-\Lambda_{(3)}\right)+\frac{1}{2} \alpha_{3}^{\vee} \cdot\left(\Lambda_{(1)}+\Lambda_{(2)}-\Lambda_{(3)}\right) \\
& -\left(-a_{1}-a_{2}+a_{6}+a_{8}-a_{9}\right) \\
& p_{1}(2,3)=\alpha_{3}^{\vee} \cdot \Lambda_{(3)}-\left(a_{1}+a_{4}+a_{5}+a_{9}\right) \\
& p_{2}(2,3)=\frac{1}{2} \alpha_{2}^{\vee} \cdot\left(-\Lambda_{(1)}+\Lambda_{(2)}+\Lambda_{(3)}\right)-\frac{1}{4}\left(\alpha_{1}^{\vee}-\alpha_{3}^{\vee}\right) \cdot\left(\Lambda_{(1)}+\Lambda_{(2)}+\Lambda_{(3)}\right) \\
& -\left(-a_{3}+a_{5}+a_{6}+a_{7}\right) \\
& p_{3}(2,3)=\frac{1}{2} \alpha_{1}^{\vee} \cdot\left(-\Lambda_{(1)}+\Lambda_{(2)}+\Lambda_{(3)}\right)+\frac{1}{2} \alpha_{3}^{\vee} \cdot\left(-\Lambda_{(1)}+\Lambda_{(2)}-\Lambda_{(3)}\right) \\
& -\left(-a_{1}+a_{3}-a_{5}-a_{7}+a_{8}\right) \\
& p_{1}(3,1)=\frac{1}{2}\left(\alpha_{1}^{\vee}+\alpha_{3}^{\vee}\right) \cdot\left(\Lambda_{(1)}-\Lambda_{(2)}+\Lambda_{(3)}\right) \\
& -\left(a_{1}+a_{2}+a_{5}+a_{7}-a_{8}+a_{9}\right) \\
& p_{2}(3,1)=\frac{1}{2} \alpha_{2}^{\vee} \cdot\left(\Lambda_{(1)}-\Lambda_{(2)}+\Lambda_{(3)}\right)-\frac{1}{4}\left(\alpha_{1}^{\vee}-\alpha_{3}^{\vee}\right) \cdot\left(\Lambda_{(1)}+\Lambda_{(2)}+\Lambda_{(3)}\right) \\
& -\left(-a_{2}+a_{4}+a_{6}+a_{8}\right) \\
& p_{3}(3,1)=a_{1} \\
& p_{1,1,2}=\frac{1}{2}\left(\alpha_{1}^{\vee}-\alpha_{3}^{\vee}\right) \cdot\left(\Lambda_{(1)}+\Lambda_{(2)}+\Lambda_{(3)}\right) \\
& -\left(a_{2}+a_{3}-a_{4}-a_{5}-a_{6}\right) \\
& p_{1,2,1}=a_{2} \quad, \quad p_{2,1,1}=a_{3} \\
& p_{2,3,3}=a_{4} \quad, \quad p_{3,2,3}=a_{5} \quad, \quad p_{3,3,2}=a_{6} \\
& p_{4,2,2}=a_{7} \quad, \quad p_{2,4,2}=a_{8} \quad, \quad p_{2,2,4}=a_{9}
\end{aligned}
$$

The generalized anharmonic ratios are readily seen to be

$$
\begin{aligned}
& A_{1}=\frac{R^{3}\left(x_{1}, x_{2}\right) R^{3}\left(x_{2}, x_{3}\right) R^{3}\left(x_{3}, x_{1}\right)}{R^{1}\left(x_{1}, x_{2}\right) R^{1}\left(x_{2}, x_{3}\right) R^{1}\left(x_{3}, x_{1}\right)} \\
& A_{2}=\frac{R^{3}\left(x_{1}, x_{2}\right) R^{2}\left(x_{3}, x_{1}\right) R^{1,2,1}\left(x_{1}, x_{2}, x_{3}\right)}{R^{2}\left(x_{1}, x_{2}\right) R^{1}\left(x_{3}, x_{1}\right) R^{1,1,2}\left(x_{1}, x_{2}, x_{3}\right)} \\
& A_{3}=\frac{R^{1}\left(x_{1}, x_{2}\right) R^{2}\left(x_{2}, x_{3}\right) R^{2,1,1}\left(x_{1}, x_{2}, x_{3}\right)}{R^{2}\left(x_{1}, x_{2}\right) R^{3}\left(x_{2}, x_{3}\right) R^{1,1,2}\left(x_{1}, x_{2}, x_{3}\right)} \\
& A_{4}=\frac{R^{1,1,2}\left(x_{1}, x_{2}, x_{3}\right) R^{2,3,3}\left(x_{1}, x_{2}, x_{3}\right)}{R^{1}\left(x_{1}, x_{2}\right) R^{1}\left(x_{2}, x_{3}\right) R^{2}\left(x_{3}, x_{1}\right)}
\end{aligned}
$$




$$
\begin{aligned}
& A_{5}=\frac{R^{3}\left(x_{2}, x_{3}\right) R^{1,1,2}\left(x_{1}, x_{2}, x_{3}\right) R^{3,2,3}\left(x_{1}, x_{2}, x_{3}\right)}{R^{1}\left(x_{1}, x_{2}\right) R^{1}\left(x_{2}, x_{3}\right) R^{2}\left(x_{2}, x_{3}\right) R^{1}\left(x_{3}, x_{1}\right)} \\
& A_{6}=\frac{R^{2}\left(x_{1}, x_{2}\right) R^{1,1,2}\left(x_{1}, x_{2}, x_{3}\right) R^{3,3,2}\left(x_{1}, x_{2}, x_{3}\right)}{R^{1}\left(x_{1}, x_{2}\right) R^{3}\left(x_{1}, x_{2}\right) R^{2}\left(x_{2}, x_{3}\right) R^{2}\left(x_{3}, x_{1}\right)} \\
& A_{7}=\frac{R^{3}\left(x_{2}, x_{3}\right) R^{4,2,2}\left(x_{1}, x_{2}, x_{3}\right)}{R^{1}\left(x_{1}, x_{2}\right) R^{2}\left(x_{2}, x_{3}\right) R^{1}\left(x_{3}, x_{1}\right)} \\
& A_{8}=\frac{R^{1}\left(x_{3}, x_{1}\right) R^{2,4,2}\left(x_{1}, x_{2}, x_{3}\right)}{R^{3}\left(x_{1}, x_{2}\right) R^{3}\left(x_{2}, x_{3}\right) R^{2}\left(x_{3}, x_{1}\right)} \\
& A_{9}=\frac{R^{3}\left(x_{1}, x_{2}\right) R^{2,2,4}\left(x_{1}, x_{2}, x_{3}\right)}{R^{2}\left(x_{1}, x_{2}\right) R^{1}\left(x_{2}, x_{3}\right) R^{1}\left(x_{3}, x_{1}\right)}
\end{aligned}
$$

Due to the syzygies listed in Appendix A, the generalized anharmonic ratios satisfy the following set of non-trivial algebraic relations

$$
\begin{aligned}
& 0=A_{7} A_{8}+t_{1} A_{6}+u_{1} \\
& 0=A_{8} A_{9}+t_{2} A_{3} A_{4}+u_{2} \\
& 0=A_{7} A_{9}+t_{3} A_{2} A_{5}+u_{3} A_{1} \\
& 0=A_{4} A_{7}+t_{4} A_{1} A_{6}+u_{4} A_{5} \\
& 0=A_{5} A_{8}+t_{5} A_{4}+u_{5} A_{6} \\
& 0=A_{6} A_{9}+t_{6} A_{5}+u_{6} A_{4} \\
& 0=A_{9}+t_{7} A_{1} A_{3}+u_{7} A_{2} \\
& 0=A_{2} A_{8}+t_{8}+u_{8} A_{3} \\
& 0=A_{3} A_{7}+t_{9} A_{2}+u_{9} \\
& 0=A_{7}+t_{10} A_{5}+u_{10} A_{1} \\
& 0=A_{9}+t_{11} A_{2} A_{4}+u_{11} A_{1} \\
& 0=A_{8}+t_{12} A_{3} A_{6}+u_{12} \\
& 0=A_{1} A_{8}+t_{13} A_{4}+u_{13} \\
& 0=A_{9}+t_{14} A_{3} A_{5}+u_{14} \\
& 0=A_{7}+t_{15} A_{2} A_{6}+u_{15}
\end{aligned}
$$

The parameters $\left(u_{\ell}, t_{\ell}\right), \ell=1, \ldots, 15$ are given in (98) in Appendix A.

Let us illustrate the procedure by considering the case

$$
\Lambda_{(1)}=(1,2,1) \quad, \quad \Lambda_{(2)}=(1,2,1) \quad, \quad \Lambda_{(3)}=(1,2,1)
$$

One may show that the corresponding tensor product coefficient is 5 , e.g. by counting independent Berenstein-Zelevinsky triangles [12] as discussed in [4]. Thus, we expect to find a 5 dimensional solution space for the chiral blocks. Now, the condition that all exponents should be non-negative integers reduces to

$$
\begin{aligned}
& 0 \leq a_{1}, \ldots, a_{9} \\
& 0 \leq 1-a_{1}+a_{3}-a_{4}-a_{5}-a_{6}-a_{7} \\
& 0 \leq 1-a_{2}-a_{3}+a_{6}-a_{9}
\end{aligned}
$$




$$
\begin{aligned}
& 0 \leq a_{1}+a_{2}-a_{6}-a_{8}+a_{9} \\
& 0 \leq 1-a_{1}-a_{4}-a_{5}-a_{9} \\
& 0 \leq 1+a_{3}-a_{5}-a_{6}-a_{7} \\
& 0 \leq a_{1}-a_{3}+a_{5}+a_{7}-a_{8} \\
& 0 \leq 1-a_{1}-a_{2}-a_{5}-a_{7}+a_{8}-a_{9} \\
& 0 \leq 1+a_{2}-a_{4}-a_{6}-a_{8} \\
& 0 \leq-a_{2}-a_{3}+a_{4}+a_{5}+a_{6}
\end{aligned}
$$

with solution

$$
\begin{aligned}
& \left(a_{1}, a_{2}, a_{3}, a_{4}, a_{5}, a_{6}\right) \\
\in \quad & (0, \ldots, 0),(1,0, \ldots, 0),(0,0,0,1,0, \ldots, 0),(0,0,0,0,1,0,0,0,0) \\
& (0, \ldots, 0,1,0,0),(0, \ldots, 0,1),(1,0, \ldots, 0,1,0),(0,1,0,1,0, \ldots, 0) \\
& (0,1,0,0,0,1,0,0,0),(0,0,1,0,1,0, \ldots, 0),(0, \ldots, 0,1,0,0,1)\}
\end{aligned}
$$

This means that the space of chiral blocks is generated by

$$
\left\{1, A_{1}, A_{4}, A_{5}, A_{7}, A_{9}, A_{1} A_{8}, A_{2} A_{4}, A_{2} A_{6}, A_{3} A_{5}, A_{6} A_{9}\right\}
$$

However, due to $(79)$ this set may be reduced to

$$
\left\{1, A_{1}, A_{4}, A_{7}, A_{9}\right\}
$$

which agrees with the tensor product coefficient being 5. In conclusion, the space of chiral blocks is 5 dimensional and is spanned by

$$
\begin{aligned}
& W_{3}^{a f f, 1}=R^{1}\left(x_{1}, x_{2}\right) R^{2}\left(x_{1}, x_{2}\right) R^{1}\left(x_{2}, x_{3}\right) R^{2}\left(x_{2}, x_{3}\right) R^{1}\left(x_{3}, x_{1}\right) R^{2}\left(x_{3}, x_{1}\right) \\
& W_{3}^{a f f, 2}=R^{2}\left(x_{1}, x_{2}\right) R^{3}\left(x_{1}, x_{2}\right) R^{2}\left(x_{2}, x_{3}\right) R^{3}\left(x_{2}, x_{3}\right) R^{2}\left(x_{3}, x_{1}\right) R^{3}\left(x_{3}, x_{1}\right) \\
& W_{3}^{a f f, 3}=R^{2}\left(x_{1}, x_{2}\right) R^{2}\left(x_{2}, x_{3}\right) R^{1}\left(x_{3}, x_{1}\right) R^{1,1,2}\left(x_{1}, x_{2}, x_{3}\right) R^{2,2,3}\left(x_{1}, x_{2}, x_{3}\right) \\
& W_{3}^{a f f, 4}=R^{2}\left(x_{1}, x_{2}\right) R^{1}\left(x_{2}, x_{3}\right) R^{3}\left(x_{2}, x_{3}\right) R^{2}\left(x_{3}, x_{1}\right) R^{4,2,2}\left(x_{1}, x_{2}, x_{3}\right) \\
& W_{3}^{a f f, 5}=R^{1}\left(x_{1}, x_{2}\right) R^{3}\left(x_{1}, x_{2}\right) R^{2}\left(x_{2}, x_{3}\right) R^{2}\left(x_{3}, x_{1}\right) R^{2,2,4}\left(x_{1}, x_{2}, x_{3}\right)
\end{aligned}
$$

From a purely algebraic point of view, it is sufficient to consider $A_{1}, A_{4}$ and $A_{7}$ as independent building block functions since the remaining 6 anharmonic ratios in addition to the constant function may be expressed in terms of these 3 by employing (79). As an illustration we find

$$
A_{6}=-\frac{1}{t_{4}} \frac{A_{4} A_{7}}{A_{1}}+\frac{u_{4}}{t_{4} t_{10}}\left(u_{10}+\frac{A_{7}}{A_{1}}\right)
$$

This observation indicates that an alternative but related procedure exists for producing 3-point functions in the case of $S L(4)$. Even though it is based on 3 building block functions (anharmonic ratios) only, it seems less transparent. In the work [5 by Rühl a procedure of that kind is discussed. It is designed explicitly to cover $S L(N)$ and he finds that the number of building block functions (in addition to equivalences to the 
elementary 2-point polynomials $\left.R^{i}\left(x_{j}, x_{k}\right)\right)$ is equal to the number of solutions to the constraint system

$$
p_{1}, p_{2}, p_{3} \in \mathbb{Z} \quad, \quad 1 \leq p_{1}, p_{2}, p_{3} \leq N \quad, \quad p_{1}+p_{2}+p_{3}=N
$$

Indeed, in the case of $S L(4)$ this number is 3. Let us emphasize that [5] only covers 2-point and 3-point functions for $S L(N)$ and that examples of 3-point functions are only worked out for $S L(3)$.

\section{Conclusion}

We shall refrain from repeating the results presented in this paper pertaining to integrable representation. Instead, we shall comment on possible generalizations.

Compared to the case of integrable representations, very little is known about nonintegrable representations. Nevertheless, degenerate [13] and in particular admissible representations [14] appear naturally in certain physical applications. An important example is provided by Hamiltonian reduction where generalized ( $W$ extended) minimal models in CFT are obtained by constraining affine current algebras for admissible representations. The entire regime of the Coulomb gas picture is obtained by considering the broader class of degenerate representations. Thus, it is of great interest to discuss generalizations from integrable to non-integrable representations. Hamiltonian reduction at the level of correlators has been discussed in [7, 15, 16]

We believe that the framework of wave functions employed in this paper (and in [6, 1]) is suitable for considering this troublesome generalization. Our belief is mainly based on the fundamental property of the formalism that all questions for integrable representations are translated into the simple language of finite polynomials and derivatives thereof. A natural proposal is to allow for finite products of monomials in the elementary polynomials raised to non-integer powers given by the generically non-integer Dynkin labels. From the mathematical point of view, such a situation is equally well under control, though some non-trivial questions arise, e.g. the dimensionality of the solution space for the chiral blocks. In the case of 2-point functions the generalization is straightforward and discussed in [1], whereas the case of 3-point functions and eventually higher point functions are currently under investigation. Also generalizations to supergroups are currently being investigated where the analogous wave function picture is described in 17 .

Furthermore, we hope to come back elsewhere with a discussion on higher point functions for integrable representations, in particular 4-point functions. Hamiltonian reduction will then yield valuable new results in Toda theory.

\section{Acknowledgment}

The author thanks Jens Schnittger for fruitful discussions and Pierre Mathieu for fruitful correspondence and for pointing out reference [2]. He gratefully acknowledges the financial support from the Danish Natural Science Research Council, contract no. 9700517. 


\section{A Examples of Elementary Polynomials and Their Associated Syzygies}

\section{A.1 Case of $S O(5)$}

We find the following 12 elementary polynomials

$$
\begin{aligned}
& R^{\Lambda^{1}, \Lambda^{1}, 0}=-2 x_{2}^{\theta} x_{2}^{1}-2\left(x_{2}^{11}\right)^{2}+\frac{1}{6}\left(x_{2}^{1} x_{2}^{2}\right)^{2}-x_{1}^{1}\left(-2 x_{2}^{\theta}+2 x_{2}^{11} x_{2}^{2}+\frac{2}{3} x_{2}^{1}\left(x_{2}^{2}\right)^{2}\right) \\
& +\left(2 x_{1}^{11}+x_{1}^{1} x_{1}^{2}\right)\left(2 x_{2}^{11}+x_{2}^{1} x_{2}^{2}\right)-\left(-2 x_{1}^{\theta}+2 x_{1}^{11} x_{1}^{2}+\frac{2}{3} x_{1}^{1}\left(x_{1}^{2}\right)^{2}\right) x_{2}^{1} \\
& -2 x_{1}^{\theta} x_{1}^{1}-2\left(x_{1}^{11}\right)^{2}+\frac{1}{6}\left(x_{1}^{1} x_{1}^{2}\right)^{2} \\
& R^{\Lambda^{2}, \Lambda^{2}, 0}=x_{2}^{\theta}+\frac{1}{6} x_{2}^{1}\left(x_{2}^{2}\right)^{2}-x_{1}^{2}\left(\frac{1}{2} x_{2}^{1} x_{2}^{2}-x_{2}^{11}\right)+\left(\frac{1}{2} x_{1}^{1} x_{1}^{2}-x_{1}^{11}\right) x_{2}^{2}-x_{1}^{\theta}-\frac{1}{6} x_{1}^{1}\left(x_{1}^{2}\right)^{2} \\
& R^{\Lambda^{1}, \Lambda^{2}, \Lambda^{2}}=\left(x_{2}^{1} x_{2}^{2}-2 x_{2}^{11}\right)\left(x_{3}^{\theta}+\frac{1}{6} x_{3}^{1}\left(x_{3}^{2}\right)^{2}\right)-\left(x_{2}^{\theta}+\frac{1}{6} x_{2}^{1}\left(x_{2}^{2}\right)^{2}\right)\left(x_{3}^{1} x_{3}^{2}-2 x_{3}^{11}\right) \\
& +2 x_{1}^{1}\left(x_{2}^{\theta}+\frac{1}{6} x_{2}^{1}\left(x_{2}^{2}\right)^{2}\right) x_{3}^{2}-2 x_{1}^{1} x_{2}^{2}\left(x_{3}^{\theta}+\frac{1}{6} x_{3}^{1}\left(x_{3}^{2}\right)^{2}\right) \\
& +\left(-x_{1}^{\theta}+x_{1}^{11} x_{1}^{2}+\frac{1}{3} x_{1}^{1}\left(x_{1}^{2}\right)^{2}\right)\left(x_{2}^{1} x_{2}^{2}-2 x_{2}^{11}\right) \\
& -\left(-x_{1}^{\theta}+x_{1}^{11} x_{1}^{2}+\frac{1}{3} x_{1}^{1}\left(x_{1}^{2}\right)^{2}\right)\left(x_{3}^{1} x_{3}^{2}-2 x_{3}^{11}\right) \\
& +\left(-2 x_{1}^{\theta} x_{1}^{1}-2\left(x_{1}^{11}\right)^{2}+\frac{1}{6}\left(x_{1}^{1} x_{1}^{2}\right)^{2}\right) x_{3}^{2} \\
& -\left(-2 x_{1}^{\theta} x_{1}^{1}-2 x_{1}^{11} x_{1}^{11}+\frac{1}{6}\left(x_{1}^{1} x_{1}^{2}\right)^{2}\right) x_{2}^{2} \\
& +\left(2 x_{1}^{11}+x_{1}^{1} x_{1}^{2}\right)\left(x_{3}^{\theta}+\frac{1}{6} x_{3}^{1}\left(x_{3}^{2}\right)^{2}\right)-\left(2 x_{1}^{11}+x_{1}^{1} x_{1}^{2}\right)\left(x_{2}^{\theta}+\frac{1}{6} x_{2}^{1}\left(x_{2}^{2}\right)^{2}\right) \\
& +\left(x_{1}^{11}+\frac{1}{3} x_{1}^{1}\left(x_{1}^{2}\right)^{2}\right) x_{2}^{2}\left(x_{3}^{1} x_{3}^{2}-2 x_{3}^{11}\right)-\left(2 x_{1}^{11}+x_{1}^{1} x_{1}^{2}\right)\left(\frac{1}{2} x_{2}^{1} x_{2}^{2}-x_{2}^{11}\right) x_{3}^{2} \\
& R^{2 \Lambda^{2}, \Lambda^{1}, \Lambda^{1}}=2\left(-2 x_{2}^{\theta}+2 x_{2}^{11}\left(x_{2}^{2}\right)^{2}+\frac{2}{3} x_{2}^{1}\left(x_{2}^{2}\right)^{2}\right)\left(-2 x_{3}^{\theta} x_{3}^{1}-2\left(x_{3}^{11}\right)^{2}+\frac{1}{6}\left(x_{3}^{1} x_{3}^{2}\right)^{2}\right) \\
& -2\left(-2 x_{2}^{\theta} x_{2}^{1}-2\left(x_{2}^{11}\right)^{2}+\frac{1}{6}\left(x_{2}^{1} x_{2}^{2}\right)^{2}\right)\left(-2 x_{3}^{\theta}+2 x_{3}^{11} x_{3}^{2}+\frac{2}{3} x_{3}^{1}\left(x_{3}^{2}\right)^{2}\right) \\
& +4 x_{1}^{2}\left(-2 x_{2}^{\theta} x_{2}^{1}-2\left(x_{2}^{11}\right)^{2}+\frac{1}{6}\left(x_{2}^{1} x_{2}^{2}\right)^{2}\right)\left(2 x_{3}^{11}+x_{3}^{1} x_{3}^{2}\right) \\
& -4 x_{1}^{2}\left(2 x_{2}^{11}+x_{2}^{1} x_{2}^{2}\right)\left(-2 x_{3}^{\theta} x_{3}^{1}-2\left(x_{3}^{11}\right)^{2}+\frac{1}{6}\left(x_{3}^{1} x_{3}^{2}\right)^{2}\right) \\
& +2\left(x_{1}^{1} x_{1}^{2}-2 x_{1}^{11}\right)\left(2 x_{2}^{11}+x_{2}^{1} x_{2}^{2}\right)\left(-2 x_{3}^{\theta}+2 x_{3}^{11} x_{3}^{2}+\frac{2}{3} x_{3}^{1}\left(x_{3}^{2}\right)^{2}\right) \\
& -2\left(x_{1}^{1} x_{1}^{2}-2 x_{1}^{11}\right)\left(-2 x_{2}^{\theta}+2 x_{2}^{11} x_{2}^{2}+\frac{2}{3} x_{2}^{1}\left(x_{2}^{2}\right)^{2}\right)\left(2 x_{3}^{11}+x_{3}^{1} x_{3}^{2}\right) \\
& +4\left(x_{1}^{2}\right)^{2} x_{2}^{1}\left(-2 x_{3}^{\theta} x_{3}^{1}-2\left(x_{3}^{11}\right)^{2}+\frac{1}{6}\left(x_{3}^{1} x_{3}^{2}\right)^{2}\right)
\end{aligned}
$$




$$
\begin{aligned}
& -4 x_{1}^{2} x_{1}^{2}\left(-2 x_{2}^{\theta} x_{2}^{1}-2\left(x_{2}^{11}\right)^{2}+\frac{1}{6}\left(x_{2}^{1} x_{2}^{2}\right)^{2}\right) x_{3}^{1} \\
& +2\left(4 x_{1}^{2} x_{1}^{\theta}+\frac{2}{3} x_{1}^{1}\left(x_{1}^{2}\right)^{3}\right)\left(x_{2}^{1}\left(2 x_{3}^{11}+x_{3}^{1} x_{3}^{2}\right)-\left(2 x_{2}^{11}+x_{2}^{1} x_{2}^{2}\right) x_{3}^{1}\right) \\
& +\quad\left(-4 x_{1}^{1} x_{1}^{2} x_{1}^{11}+4\left(x_{1}^{11}\right)^{2}+\left(x_{1}^{1} x_{1}^{2}\right)^{2}\right) \\
& \cdot \quad\left(2 x_{2}^{\theta}-2 x_{2}^{11} x_{2}^{2}-\frac{2}{3} x_{2}^{1}\left(x_{2}^{2}\right)^{2}-2 x_{3}^{\theta}+2 x_{3}^{11} x_{3}^{2}+\frac{2}{3} x_{3}^{1}\left(x_{3}^{2}\right)^{2}\right) \\
& +\quad\left(4 x_{1}^{\theta} x_{1}^{1} x_{1}^{2}-8 x_{1}^{\theta} x_{1}^{11}-\frac{4}{3} x_{1}^{1}\left(x_{1}^{2}\right)^{2} x_{1}^{11}+\frac{2}{3}\left(x_{1}^{1}\right)^{2}\left(x_{1}^{2}\right)^{3}\right) \\
& \cdot \quad\left(2 x_{2}^{11}+x_{2}^{1} x_{2}^{2}-2 x_{3}^{11}-x_{3}^{1} x_{3}^{2}\right) \\
& +\quad\left(8\left(x_{1}^{\theta}\right)^{2}-\frac{4}{3} x_{1}^{1}\left(x_{1}^{2}\right)^{3} x_{1}^{11}+\frac{8}{3} x_{1}^{1}\left(x_{1}^{2}\right)^{2} x_{1}^{\theta}-\frac{4}{9}\left(x_{1}^{1}\right)^{2}\left(x_{1}^{2}\right)^{4}\right)\left(x_{3}^{1}-x_{2}^{1}\right) \\
& +\quad 4 x_{1}^{2}\left(x_{1}^{1} x_{1}^{2}-2 x_{1}^{11}\right) \\
& \cdot \quad\left(-2 x_{2}^{\theta} x_{2}^{1}-2\left(x_{2}^{11}\right)^{2}+\frac{1}{6}\left(x_{2}^{1} x_{2}^{2}\right)^{2}+2 x_{3}^{\theta} x_{3}^{1}-2\left(x_{3}^{11}\right)^{2}+\frac{1}{6}\left(x_{3}^{1} x_{3}^{2}\right)^{2}\right) \\
& +\quad 2\left(2 x_{1}^{\theta}-2 x_{1}^{11} x_{1}^{2}+\frac{4}{3} x_{1}^{1}\left(x_{1}^{2}\right)^{2}\right) \\
& \quad\left\{2 x_{2}^{\theta} x_{2}^{1}+2\left(x_{2}^{11}\right)^{2}-\frac{1}{6}\left(x_{2}^{1} x_{2}^{2}\right)^{2}-2 x_{3}^{\theta} x_{3}^{1}-2\left(x_{3}^{11}\right)^{2}+\frac{1}{6}\left(x_{3}^{1} x_{3}^{2}\right)^{2}\right. \\
& \left.+\quad\left(-2 x_{2}^{\theta}+2 x_{2}^{11} x_{2}^{2}+\frac{2}{3} x_{2}^{1}\left(x_{2}^{2}\right)^{2}\right) x_{3}^{1}-x_{2}^{1}\left(-2 x_{3}^{\theta}+2 x_{3}^{11} x_{3}^{2}+\frac{2}{3} x_{3}^{1}\left(x_{3}^{2}\right)^{2}\right)\right\} \\
& +
\end{aligned}
$$

including the "permuted" ones

$$
\begin{aligned}
R^{\Lambda^{1}, 0, \Lambda^{1}}\left(x_{1}, x_{2}, x_{3}\right) & =R^{\Lambda^{1}, \Lambda^{1}, 0}\left(x_{1}, x_{3}, x_{2}\right) \\
R^{0, \Lambda^{1}, \Lambda^{1}}\left(x_{1}, x_{2}, x_{3}\right) & =R^{\Lambda^{1}, \Lambda^{1}, 0}\left(x_{2}, x_{3}, x_{1}\right) \\
R^{\Lambda^{2}, 0, \Lambda^{2}}\left(x_{1}, x_{2}, x_{3}\right) & =R^{\Lambda^{2}, \Lambda^{2}, 0}\left(x_{1}, x_{3}, x_{2}\right) \\
R^{0, \Lambda^{2}, \Lambda^{2}}\left(x_{1}, x_{2}, x_{3}\right) & =R^{\Lambda^{2}, \Lambda^{2}, 0}\left(x_{2}, x_{3}, x_{1}\right) \\
R^{\Lambda^{2}, \Lambda^{1}, \Lambda^{2}}\left(x_{1}, x_{2}, x_{3}\right) & =R^{\Lambda^{1}, \Lambda^{2}, \Lambda^{2}}\left(x_{2}, x_{1}, x_{3}\right) \\
R^{\Lambda^{2}, \Lambda^{2}, \Lambda^{1}}\left(x_{1}, x_{2}, x_{3}\right) & =R^{\Lambda^{1}, \Lambda^{2}, \Lambda^{2}}\left(x_{3}, x_{2}, x_{1}\right) \\
R^{\Lambda^{1}, 2 \Lambda^{2}, \Lambda^{1}}\left(x_{1}, x_{2}, x_{3}\right) & =R^{2 \Lambda^{2}, \Lambda^{1}, \Lambda^{1}}\left(x_{2}, x_{1}, x_{3}\right) \\
R^{\Lambda^{1}, \Lambda^{1}, 2 \Lambda^{2}}\left(x_{1}, x_{2}, x_{3}\right) & =R^{2 \Lambda^{2}, \Lambda^{1}, \Lambda^{1}}\left(x_{3}, x_{2}, x_{1}\right)
\end{aligned}
$$

In (88) the order of the arguments are $R\left(x_{1}, x_{2}, x_{3}\right)$. In terms of these elementary polynomials the 9 syzygies become

$$
\begin{aligned}
0 & =s_{1} R^{0, \Lambda^{2}, \Lambda^{2}}\left(x_{1}, x_{2}, x_{3}\right) R^{2 \Lambda^{2}, \Lambda^{1}, \Lambda^{1}}\left(x_{1}, x_{2}, x_{3}\right) \\
& +t_{1} R^{\Lambda^{2}, \Lambda^{1}, \Lambda^{2}}\left(x_{1}, x_{2}, x_{3}\right) R^{\Lambda^{2}, \Lambda^{2}, \Lambda^{1}}\left(x_{1}, x_{2}, x_{3}\right) \\
& +u_{1} R^{0, \Lambda^{1}, \Lambda^{1}}\left(x_{1}, x_{2}, x_{3}\right) R^{\Lambda^{2}, \Lambda^{2}, 0}\left(x_{1}, x_{2}, x_{3}\right) R^{\Lambda^{2}, 0, \Lambda^{2}}\left(x_{1}, x_{2}, x_{3}\right) \\
0 & =s_{2} R^{\Lambda^{2}, 0, \Lambda^{2}}\left(x_{1}, x_{2}, x_{3}\right) R^{\Lambda^{1}, 2 \Lambda^{2}, \Lambda^{1}}\left(x_{1}, x_{2}, x_{3}\right) \\
& +t_{2} R^{\Lambda^{1}, \Lambda^{2}, \Lambda^{2}}\left(x_{1}, x_{2}, x_{3}\right) R^{\Lambda^{2}, \Lambda^{2}, \Lambda^{1}}\left(x_{1}, x_{2}, x_{3}\right) \\
& +u_{2} R^{\Lambda^{1}, 0, \Lambda^{1}}\left(x_{1}, x_{2}, x_{3}\right) R^{\Lambda^{2}, \Lambda^{2}, 0}\left(x_{1}, x_{2}, x_{3}\right) R^{0, \Lambda^{2}, \Lambda^{2}}\left(x_{1}, x_{2}, x_{3}\right)
\end{aligned}
$$




$$
\begin{aligned}
& 0=s_{3} R^{\Lambda^{2}, \Lambda^{2}, 0}\left(x_{1}, x_{2}, x_{3}\right) R^{\Lambda^{1}, \Lambda^{1}, 2 \Lambda^{2}}\left(x_{1}, x_{2}, x_{3}\right) \\
& +t_{3} R^{\Lambda^{1}, \Lambda^{2}, \Lambda^{2}}\left(x_{1}, x_{2}, x_{3}\right) R^{\Lambda^{2}, \Lambda^{1}, \Lambda^{2}}\left(x_{1}, x_{2}, x_{3}\right) \\
& +u_{3} R^{\Lambda^{1}, \Lambda^{1}, 0}\left(x_{1}, x_{2}, x_{3}\right) R^{\Lambda^{2}, 0, \Lambda^{2}}\left(x_{1}, x_{2}, x_{3}\right) R^{0, \Lambda^{2}, \Lambda^{2}}\left(x_{1}, x_{2}, x_{3}\right) \\
& 0=s_{4} R^{\Lambda^{1}, \Lambda^{2}, \Lambda^{2}}\left(x_{1}, x_{2}, x_{3}\right) R^{2 \Lambda^{2}, \Lambda^{1}, \Lambda^{1}}\left(x_{1}, x_{2}, x_{3}\right) \\
& +t_{4} R^{\Lambda^{1}, 0, \Lambda^{1}}\left(x_{1}, x_{2}, x_{3}\right) R^{\Lambda^{2}, \Lambda^{2}, 0}\left(x_{1}, x_{2}, x_{3}\right) R^{\Lambda^{2}, \Lambda^{1}, \Lambda^{2}}\left(x_{1}, x_{2}, x_{3}\right) \\
& +u_{4} R^{\Lambda^{1}, \Lambda^{1}, 0}\left(x_{1}, x_{2}, x_{3}\right) R^{\Lambda^{2}, 0, \Lambda^{2}}\left(x_{1}, x_{2}, x_{3}\right) R^{\Lambda^{2}, \Lambda^{2}, \Lambda^{1}}\left(x_{1}, x_{2}, x_{3}\right) \\
& 0=s_{5} R^{\Lambda^{2}, \Lambda^{1}, \Lambda^{2}}\left(x_{1}, x_{2}, x_{3}\right) R^{\Lambda^{1}, 2 \Lambda^{2}, \Lambda^{1}}\left(x_{1}, x_{2}, x_{3}\right) \\
& +t_{5} R^{0, \Lambda^{1}, \Lambda^{1}}\left(x_{1}, x_{2}, x_{3}\right) R^{\Lambda^{2}, \Lambda^{2}, 0}\left(x_{1}, x_{2}, x_{3}\right) R^{\Lambda^{1}, \Lambda^{2}, \Lambda^{2}}\left(x_{1}, x_{2}, x_{3}\right) \\
& +u_{5} R^{\Lambda^{1}, \Lambda^{1}, 0}\left(x_{1}, x_{2}, x_{3}\right) R^{0, \Lambda^{2}, \Lambda^{2}}\left(x_{1}, x_{2}, x_{3}\right) R^{\Lambda^{2}, \Lambda^{2}, \Lambda^{1}}\left(x_{1}, x_{2}, x_{3}\right) \\
& 0=s_{6} R^{\Lambda^{2}, \Lambda^{2}, \Lambda^{1}}\left(x_{1}, x_{2}, x_{3}\right) R^{\Lambda^{1}, \Lambda^{1}, 2 \Lambda^{2}}\left(x_{1}, x_{2}, x_{3}\right) \\
& +t_{6} R^{0, \Lambda^{1}, \Lambda^{1}}\left(x_{1}, x_{2}, x_{3}\right) R^{\Lambda^{2}, 0, \Lambda^{2}}\left(x_{1}, x_{2}, x_{3}\right) R^{\Lambda^{1}, \Lambda^{2}, \Lambda^{2}}\left(x_{1}, x_{2}, x_{3}\right) \\
& +u_{6} R^{\Lambda^{1}, 0, \Lambda^{1}}\left(x_{1}, x_{2}, x_{3}\right) R^{0, \Lambda^{2}, \Lambda^{2}}\left(x_{1}, x_{2}, x_{3}\right) R^{\Lambda^{2}, \Lambda^{1}, \Lambda^{2}}\left(x_{1}, x_{2}, x_{3}\right) \\
& 0=s_{7} R^{2 \Lambda^{2}, \Lambda^{1}, \Lambda^{1}}\left(x_{1}, x_{2}, x_{3}\right) R^{\Lambda^{1}, 2 \Lambda^{2}, \Lambda^{1}}\left(x_{1}, x_{2}, x_{3}\right) \\
& +t_{7} R^{\Lambda^{1}, \Lambda^{1}, 0}\left(x_{1}, x_{2}, x_{3}\right) R^{\Lambda^{2}, \Lambda^{2}, \Lambda^{1}}\left(x_{1}, x_{2}, x_{3}\right) R^{\Lambda^{2}, \Lambda^{2}, \Lambda^{1}}\left(x_{1}, x_{2}, x_{3}\right) \\
& +u_{7} R^{\Lambda^{1}, 0, \Lambda^{1}}\left(x_{1}, x_{2}, x_{3}\right) R^{0, \Lambda^{1}, \Lambda^{1}}\left(x_{1}, x_{2}, x_{3}\right) R^{\Lambda^{2}, \Lambda^{2}, 0}\left(x_{1}, x_{2}, x_{3}\right) R^{\Lambda^{2}, \Lambda^{2}, 0}\left(x_{1}, x_{2}, x_{3}\right) \\
& 0=s_{8} R^{2 \Lambda^{2}, \Lambda^{1}, \Lambda^{1}}\left(x_{1}, x_{2}, x_{3}\right) R^{\Lambda^{1}, \Lambda^{1}, 2 \Lambda^{2}}\left(x_{1}, x_{2}, x_{3}\right) \\
& +t_{8} R^{\Lambda^{1}, 0, \Lambda^{1}}\left(x_{1}, x_{2}, x_{3}\right) R^{\Lambda^{2}, \Lambda^{1}, \Lambda^{2}}\left(x_{1}, x_{2}, x_{3}\right) R^{\Lambda^{2}, \Lambda^{1}, \Lambda^{2}}\left(x_{1}, x_{2}, x_{3}\right) \\
& +u_{8} R^{\Lambda^{1}, \Lambda^{1}, 0}\left(x_{1}, x_{2}, x_{3}\right) R^{0, \Lambda^{1}, \Lambda^{1}}\left(x_{1}, x_{2}, x_{3}\right) R^{\Lambda^{2}, 0, \Lambda^{2}}\left(x_{1}, x_{2}, x_{3}\right) R^{\Lambda^{2}, 0, \Lambda^{2}}\left(x_{1}, x_{2}, x_{3}\right) \\
& 0=s_{9} R^{\Lambda^{1}, 2 \Lambda^{2}, \Lambda^{1}}\left(x_{1}, x_{2}, x_{3}\right) R^{\Lambda^{1}, \Lambda^{1}, 2 \Lambda^{2}}\left(x_{1}, x_{2}, x_{3}\right) \\
& +t_{9} R^{0, \Lambda^{1}, \Lambda^{1}}\left(x_{1}, x_{2}, x_{3}\right) R^{\Lambda^{1}, \Lambda^{2}, \Lambda^{2}}\left(x_{1}, x_{2}, x_{3}\right) R^{\Lambda^{1}, \Lambda^{2}, \Lambda^{2}}\left(x_{1}, x_{2}, x_{3}\right) \\
& +u_{9} R^{\Lambda^{1}, \Lambda^{1}, 0}\left(x_{1}, x_{2}, x_{3}\right) R^{\Lambda^{1}, 0, \Lambda^{1}}\left(x_{1}, x_{2}, x_{3}\right) R^{0, \Lambda^{2}, \Lambda^{2}}\left(x_{1}, x_{2}, x_{3}\right) R^{0, \Lambda^{2}, \Lambda^{2}}\left(x_{1}, x_{2}, x_{3}\right)(90)
\end{aligned}
$$

where the first 3 correspond to the coupling $\left(2 \Lambda^{2}, \Lambda^{1}+\Lambda^{2}, \Lambda^{1}+\Lambda^{2}\right)$ and permutations thereof, the next 3 correspond to $\left(\Lambda^{1}+2 \Lambda^{2}, \Lambda^{1}+\Lambda^{2}, \Lambda^{1}+\Lambda^{2}\right)$ and permutations thereof, while the last 3 correspond to $\left(\Lambda^{1}+2 \Lambda^{2}, \Lambda^{1}+2 \Lambda^{2}, 2 \Lambda^{1}\right)$ and permutations thereof. In the normalization chosen, (88) and (89), the parameters are worked out to be

$$
\begin{aligned}
& \left(t_{1}, u_{1}\right)=(2,4) \\
& \left(t_{2}, u_{2}\right)=(-2,-4) \\
& \left(t_{5}, u_{5}\right)=(4,-4) \\
& \left(t_{7}, u_{7}\right)=(8,16) \\
& \left(t_{8}, u_{8}\right)=(8,16) \\
& \begin{aligned}
\left(t_{3}, u_{3}\right) & =(2,-4) \\
\left(t_{6}, u_{6}\right) & =(-4,-4) \\
\left(t_{9}, u_{9}\right) & =(-8,-16)
\end{aligned}
\end{aligned}
$$

where we have fixed $s_{\ell}=1, \ell=1, \ldots, 9$.

\section{A.2 Case of $S L(4)$}

We find that the following polynomials constitute the wave function equivalences of basis vectors in the 4 highest weight modules $\Lambda^{1}, \Lambda^{2}, \Lambda^{3}$ and $\Lambda^{1}+\Lambda^{3}$, respectively

$$
\begin{aligned}
K_{100}(x) & =1 \\
K_{-110}(x) & =x^{1}
\end{aligned}
$$




$$
\begin{aligned}
K_{0-11}(x) & =x^{12}+\frac{1}{2} x^{1} x^{2} \\
K_{00-1}(x) & =x^{\theta}+\frac{1}{2} x^{12} x^{3}+\frac{1}{2} x^{1} x^{23}+\frac{1}{6} x^{1} x^{2} x^{3} \\
K_{010} & =1 \\
K_{1-11}(x) & =x^{2} \\
K_{-101}(x) & =-x^{12}+\frac{1}{2} x^{1} x^{2} \\
K_{10-1}(x) & =x^{23}+\frac{1}{2} x^{2} x^{3} \\
K_{-11-1}(x) & =-x^{\theta}+\frac{1}{2} x^{1} x^{23}-\frac{1}{2} x^{12} x^{3}+\frac{1}{3} x^{1} x^{2} x^{3} \\
K_{0-10}(x) & =-x^{2} x^{\theta}+x^{12} x^{23}+\frac{1}{12} x^{1}\left(x^{2}\right)^{2} x^{3} \\
K_{001}(x) & =1 \\
K_{01-1}(x) & =x^{3} \\
K_{1-10}(x) & =-x^{23}+\frac{1}{2} x^{2} x^{3} \\
K_{-100}(x) & =x^{\theta}-\frac{1}{2} x^{1} x^{23}-\frac{1}{2} x^{12} x^{3}+\frac{1}{6} x^{1} x^{2} x^{3}
\end{aligned}
$$

$$
\begin{aligned}
K_{101}(x) & =1 \\
K_{-111}(x) & =x^{1} \\
K_{11-1}(x) & =x^{3} \\
K_{0-12}(x) & =x^{12}+\frac{1}{2} x^{1} x^{2} \\
K_{2-10}(x) & =-x^{23}+\frac{1}{2} x^{2} x^{3} \\
K_{-12-1}(x) & =x^{1} x^{3} \\
L_{1}(x) & =x^{\theta}-\frac{3}{2} x^{1} x^{23}-\frac{1}{2} x^{12} x^{3}+\frac{2}{3} x^{1} x^{2} x^{3} \\
L_{2}(x) & =-x^{1} x^{23}+x^{12} x^{3}+x^{1} x^{2} x^{3} \\
L_{3}(x) & =x^{\theta}+\frac{3}{2} x^{12} x^{3}+\frac{1}{2} x^{1} x^{23}+\frac{2}{3} x^{1} x^{2} x^{3} \\
K_{-210}(x) & =2 x^{1} x^{\theta}-\left(x^{1}\right)^{2} x^{23}-x^{1} x^{12} x^{3}+\frac{1}{3}\left(x^{1}\right)^{2} x^{2} x^{3} \\
K_{01-2}(x) & =2 x^{3} x^{\theta}+x^{12}\left(x^{3}\right)^{2}+x^{1} x^{23} x^{3}+\frac{1}{3} x^{1} x^{2}\left(x^{3}\right)^{2} \\
K_{1-21}(x) & =-2 x^{12} x^{23}+x^{12} x^{2} x^{3}-x^{1} x^{2} x^{23}+\frac{1}{2} x^{1}\left(x^{2}\right)^{2} x^{3} \\
K_{1-1-1}(x) & =-2 x^{23} x^{\theta}+x^{2} x^{3} x^{\theta}-x^{12} x^{23} x^{3}-x^{1}\left(x^{23}\right)^{2}+\frac{1}{2} x^{12} x^{2}\left(x^{3}\right)^{2}
\end{aligned}
$$




$$
\begin{aligned}
& +\frac{1}{6} x^{1} x^{2} x^{23} x^{3}+\frac{1}{6} x^{1}\left(x^{2} x^{3}\right)^{2} \\
K_{-1-11}(x) & =2 x^{12} x^{\theta}+x^{1} x^{2} x^{\theta}-x^{1} x^{12} x^{23}-\left(x^{12}\right)^{2} x^{3}-\frac{1}{2}\left(x^{1}\right)^{2} x^{2} x^{23} \\
& -\frac{1}{6} x^{1} x^{12} x^{2} x^{3}+\frac{1}{6}\left(x^{1} x^{2}\right)^{2} x^{3} \\
K_{-10-1}(x) & =2\left(x^{\theta}\right)^{2}+\frac{2}{3} x^{1} x^{2} x^{3} x^{\theta}-\frac{1}{2}\left(x^{1} x^{23}\right)^{2}-\frac{1}{2}\left(x^{12} x^{3}\right)^{2} \\
& -x^{1} x^{12} x^{23} x^{3}+\frac{1}{18}\left(x^{1} x^{2} x^{3}\right)^{2}
\end{aligned}
$$

The indices denote the weights of the vectors where we have used the obvious abbreviation leaving out commas. The special notation $L_{1}, L_{2}, L_{3}$ is introduced to characterize the polynomials associated to the weight 0 which appears with multiplicity 3 in the highest weight module $\Lambda^{1}+\Lambda^{3}$. In terms of these, the 18 elementary polynomials are worked out to be

$$
\begin{aligned}
& R^{\Lambda^{1}, \Lambda^{3}, 0}\left(x_{1}, x_{2}, x_{3}\right)=K_{100}\left(x_{1}\right) K_{-100}\left(x_{2}\right)-K_{-110}\left(x_{1}\right) K_{1-10}\left(x_{2}\right) \\
& +K_{0-11}\left(x_{1}\right) K_{01-1}\left(x_{2}\right)-K_{00-1}\left(x_{1}\right) K_{001}\left(x_{2}\right) \\
& R^{\Lambda^{1}, 0, \Lambda^{3}}\left(x_{1}, x_{2}, x_{3}\right)=R^{\Lambda^{1}, \Lambda^{3}, 0}\left(x_{1}, x_{3}, x_{2}\right) \\
& R^{0, \Lambda^{1}, \Lambda^{3}}\left(x_{1}, x_{2}, x_{3}\right)=R^{\Lambda^{1}, \Lambda^{3}, 0}\left(x_{2}, x_{3}, x_{1}\right) \\
& R^{\Lambda^{3}, \Lambda^{1}, 0}\left(x_{1}, x_{2}, x_{3}\right)=R^{\Lambda^{1}, \Lambda^{3}, 0}\left(x_{2}, x_{1}, x_{3}\right) \\
& R^{\Lambda^{3}, 0, \Lambda^{1}}\left(x_{1}, x_{2}, x_{3}\right)=R^{\Lambda^{1}, \Lambda^{3}, 0}\left(x_{3}, x_{1}, x_{2}\right) \\
& R^{0, \Lambda^{3}, \Lambda^{1}}\left(x_{1}, x_{2}, x_{3}\right)=R^{\Lambda^{1}, \Lambda^{3}, 0}\left(x_{3}, x_{2}, x_{1}\right) \\
& R^{\Lambda^{2}, \Lambda^{2}, 0}\left(x_{1}, x_{2}, x_{3}\right)=K_{010}\left(x_{1}\right) K_{0-10}\left(x_{2}\right)-K_{1-11}\left(x_{1}\right) K_{-11-1}\left(x_{2}\right) \\
& +K_{-101}\left(x_{1}\right) K_{10-1}\left(x_{2}\right)+K_{10-1}\left(x_{1}\right) K_{-101}\left(x_{2}\right) \\
& \text { - } K_{-11-1}\left(x_{1}\right) K_{1-11}\left(x_{2}\right)+K_{0-10}\left(x_{1}\right) K_{010}\left(x_{2}\right) \\
& R^{0, \Lambda^{2}, \Lambda^{2}}\left(x_{1}, x_{2}, x_{3}\right)=R^{\Lambda^{2}, \Lambda^{2}, 0}\left(x_{3}, x_{2}, x_{1}\right) \\
& R^{\Lambda^{2}, 0, \Lambda^{2}}\left(x_{1}, x_{2}, x_{3}\right)=R^{\Lambda^{2}, \Lambda^{2}, 0}\left(x_{1}, x_{3}, x_{2}\right) \\
& R^{\Lambda^{2}, \Lambda^{3}, \Lambda^{3}}\left(x_{1}, x_{2}, x_{3}\right)=K_{010}\left(x_{1}\right)\left\{K_{1-10}\left(x_{2}\right) K_{-100}\left(x_{3}\right)-K_{-100}\left(x_{2}\right) K_{1-10}\left(x_{3}\right)\right\} \\
& +K_{1-11}\left(x_{1}\right)\left\{K_{-100}\left(x_{2}\right) K_{01-1}\left(x_{3}\right)-K_{01-1}\left(x_{2}\right) K_{-100}\left(x_{3}\right)\right\} \\
& +K_{-101}\left(x_{1}\right)\left\{K_{01-1}\left(x_{2}\right) K_{1-10}\left(x_{3}\right)-K_{1-10}\left(x_{2}\right) K_{01-1}\left(x_{3}\right)\right\} \\
& +K_{10-1}\left(x_{1}\right)\left\{K_{001}\left(x_{2}\right) K_{-100}\left(x_{3}\right)-K_{-100}\left(x_{2}\right) K_{001}\left(x_{3}\right)\right\} \\
& +K_{-11-1}\left(x_{1}\right)\left\{K_{1-10}\left(x_{2}\right) K_{001}\left(x_{3}\right)-K_{001}\left(x_{2}\right) K_{1-10}\left(x_{3}\right)\right\} \\
& +K_{0-10}\left(x_{1}\right)\left\{K_{001}\left(x_{2}\right) K_{01-1}\left(x_{3}\right)-K_{01-1}\left(x_{2}\right) K_{001}\left(x_{3}\right)\right\} \\
& R^{\Lambda^{3}, \Lambda^{2}, \Lambda^{3}}\left(x_{1}, x_{2}, x_{3}\right)=R^{\Lambda^{2}, \Lambda^{3}, \Lambda^{3}}\left(x_{2}, x_{1}, x_{3}\right) \\
& R^{\Lambda^{3}, \Lambda^{3}, \Lambda^{2}}\left(x_{1}, x_{2}, x_{3}\right)=R^{\Lambda^{2}, \Lambda^{3}, \Lambda^{3}}\left(x_{3}, x_{2}, x_{1}\right) \\
& R^{\Lambda^{2}, \Lambda^{1}, \Lambda^{1}}\left(x_{1}, x_{2}, x_{3}\right)=K_{010}\left(x_{1}\right)\left\{K_{0-11}\left(x_{2}\right) K_{00-1}\left(x_{3}\right)-K_{00-1}\left(x_{2}\right) K_{0-11}\left(x_{3}\right)\right\} \\
& +K_{1-11}\left(x_{1}\right)\left\{K_{00-1}\left(x_{2}\right) K_{-110}\left(x_{3}\right)-K_{-110}\left(x_{2}\right) K_{00-1}\left(x_{3}\right)\right\} \\
& +K_{-101}\left(x_{1}\right)\left\{K_{100}\left(x_{2}\right) K_{00-1}\left(x_{3}\right)-K_{00-1}\left(x_{2}\right) K_{100}\left(x_{3}\right)\right\} \\
& +K_{10-1}\left(x_{1}\right)\left\{K_{-110}\left(x_{2}\right) K_{0-11}\left(x_{3}\right)-K_{0-11}\left(x_{2}\right) K_{-110}\left(x_{3}\right)\right\}
\end{aligned}
$$




$$
\begin{aligned}
& +K_{-11-1}\left(x_{1}\right)\left\{K_{0-11}\left(x_{2}\right) K_{100}\left(x_{3}\right)-K_{100}\left(x_{2}\right) K_{0-11}\left(x_{3}\right)\right\} \\
& +K_{0-10}\left(x_{1}\right)\left\{K_{100}\left(x_{2}\right) K_{-110}\left(x_{3}\right)-K_{-110}\left(x_{2}\right) K_{100}\left(x_{3}\right)\right\} \\
& R^{\Lambda^{1}, \Lambda^{2}, \Lambda^{1}}\left(x_{1}, x_{2}, x_{3}\right)=R^{\Lambda^{2}, \Lambda^{1}, \Lambda^{1}}\left(x_{2}, x_{1}, x_{3}\right) \\
& R^{\Lambda^{1}, \Lambda^{1}, \Lambda^{2}}\left(x_{1}, x_{2}, x_{3}\right)=R^{\Lambda^{2}, \Lambda^{1}, \Lambda^{1}}\left(x_{3}, x_{2}, x_{1}\right) \\
& R^{\Lambda^{1}+\Lambda^{3}, \Lambda^{2}, \Lambda^{2}}\left(x_{1}, x_{2}, x_{3}\right)=2 K_{101}\left(x_{1}\right)\left\{K_{-11-1}\left(x_{2}\right) K_{0-10}\left(x_{3}\right)-K_{0-10}\left(x_{2}\right) K_{-11-1}\left(x_{3}\right)\right\} \\
& +2 K_{-111}\left(x_{1}\right)\left\{K_{0-10}\left(x_{2}\right) K_{10-1}\left(x_{3}\right)-K_{10-1}\left(x_{2}\right) K_{0-10}\left(x_{3}\right)\right\} \\
& +2 K_{11-1}\left(x_{1}\right)\left\{K_{0-10}\left(x_{2}\right) K_{-101}\left(x_{3}\right)-K_{-101}\left(x_{2}\right) K_{0-10}\left(x_{3}\right)\right\} \\
& +2 K_{0-12}\left(x_{1}\right)\left\{K_{10-1}\left(x_{2}\right) K_{-11-1}\left(x_{3}\right)-K_{-11-1}\left(x_{2}\right) K_{10-1}\left(x_{3}\right)\right\} \\
& +2 K_{-12-1}\left(x_{1}\right)\left\{K_{1-11}\left(x_{2}\right) K_{0-10}\left(x_{3}\right)-K_{0-10}\left(x_{2}\right) K_{1-11}\left(x_{3}\right)\right\} \\
& +2 K_{2-10}\left(x_{1}\right)\left\{K_{-101}\left(x_{2}\right) K_{-11-1}\left(x_{3}\right)-K_{-11-1}\left(x_{2}\right) K_{-101}\left(x_{3}\right)\right\} \\
& +K_{01-2}\left(x_{1}\right)\left\{K_{1-11}\left(x_{2}\right) K_{-101}\left(x_{3}\right)-K_{-101}\left(x_{2}\right) K_{1-11}\left(x_{3}\right)\right\} \\
& +K_{1-21}\left(x_{1}\right)\left\{K_{010}\left(x_{2}\right) K_{-11-1}\left(x_{3}\right)-K_{-11-1}\left(x_{2}\right) K_{010}\left(x_{3}\right)\right\} \\
& +K_{-210}\left(x_{1}\right)\left\{K_{1-11}\left(x_{2}\right) K_{10-1}\left(x_{3}\right)-K_{10-1}\left(x_{2}\right) K_{1-11}\left(x_{3}\right)\right\} \\
& +K_{1-1-1}\left(x_{1}\right)\left\{K_{-101}\left(x_{2}\right) K_{010}\left(x_{3}\right)-K_{010}\left(x_{2}\right) K_{-101}\left(x_{3}\right)\right\} \\
& +K_{-1-11}\left(x_{1}\right)\left\{K_{10-1}\left(x_{2}\right) K_{010}\left(x_{3}\right)-K_{010}\left(x_{2}\right) K_{10-1}\left(x_{3}\right)\right\} \\
& +K_{-10-1}\left(x_{1}\right)\left\{K_{010}\left(x_{2}\right) K_{1-11}\left(x_{3}\right)-K_{1-11}\left(x_{2}\right) K_{010}\left(x_{3}\right)\right\} \\
& +L_{1}\left(x_{1}\right)\left\{K_{010}\left(x_{2}\right) K_{0-10}\left(x_{3}\right)-K_{0-10}\left(x_{2}\right) K_{010}\left(x_{3}\right)\right. \\
& +K_{-11-1}\left(x_{2}\right) K_{1-11}\left(x_{3}\right)-K_{1-11}\left(x_{2}\right) K_{-11-1}\left(x_{3}\right) \\
& \left.+K_{10-1}\left(x_{2}\right) K_{-101}\left(x_{3}\right)-K_{-101}\left(x_{2}\right) K_{10-1}\left(x_{3}\right)\right\} \\
& +2 L_{2}\left(x_{1}\right)\left\{K_{0-10}\left(x_{2}\right) K_{010}\left(x_{3}\right)-K_{010}\left(x_{2}\right) K_{0-10}\left(x_{3}\right)\right\} \\
& +L_{3}\left(x_{1}\right)\left\{K_{010}\left(x_{2}\right) K_{0-10}\left(x_{3}\right)-K_{0-10}\left(x_{2}\right) K_{010}\left(x_{3}\right)\right. \\
& +K_{-11-1}\left(x_{2}\right) K_{1-11}\left(x_{3}\right)-K_{1-11}\left(x_{2}\right) K_{-11-1}\left(x_{3}\right) \\
& \left.+K_{-101}\left(x_{2}\right) K_{10-1}\left(x_{3}\right)-K_{10-1}\left(x_{2}\right) K_{-101}\left(x_{3}\right)\right\} \\
& R^{\Lambda^{2}, \Lambda^{1}+\Lambda^{3}, \Lambda^{2}}\left(x_{1}, x_{2}, x_{3}\right)=R^{\Lambda^{1}+\Lambda^{3}, \Lambda^{2}, \Lambda^{2}}\left(x_{2}, x_{1}, x_{3}\right) \\
& R^{\Lambda^{2}, \Lambda^{2}, \Lambda^{1}+\Lambda^{3}}\left(x_{1}, x_{2}, x_{3}\right)=R^{\Lambda^{1}+\Lambda^{3}, \Lambda^{2}, \Lambda^{2}}\left(x_{3}, x_{2}, x_{1}\right)
\end{aligned}
$$

while the 15 associated syzygies become

$$
\begin{aligned}
0 & =s_{1} R^{\Lambda^{1}+\Lambda^{3}, \Lambda^{2}, \Lambda^{2}}\left(x_{1}, x_{2}, x_{3}\right) R^{\Lambda^{2}, \Lambda^{1}+\Lambda^{3}, \Lambda^{2}}\left(x_{1}, x_{2}, x_{3}\right) \\
& +t_{1} R^{\Lambda^{2}, \Lambda^{2}, 0}\left(x_{1}, x_{2}, x_{3}\right) R^{\Lambda^{1}, \Lambda^{1}, \Lambda^{2}}\left(x_{1}, x_{2}, x_{3}\right) R^{\Lambda^{3}, \Lambda^{3}, \Lambda^{2}}\left(x_{1}, x_{2}, x_{3}\right) \\
& +u_{1} R^{0, \Lambda^{2}, \Lambda^{2}}\left(x_{1}, x_{2}, x_{3}\right) R^{\Lambda^{2}, 0, \Lambda^{2}}\left(x_{1}, x_{2}, x_{3}\right) R^{\Lambda^{3}, \Lambda^{1}, 0}\left(x_{1}, x_{2}, x_{3}\right) R^{\Lambda^{1}, \Lambda^{3}, 0}\left(x_{1}, x_{2}, x_{3}\right) \\
0 & =s_{2} R^{\Lambda^{2}, \Lambda^{2}, \Lambda^{1}+\Lambda^{3}}\left(x_{1}, x_{2}, x_{3}\right) R^{\Lambda^{2}, \Lambda^{1}+\Lambda^{3}, \Lambda^{2}}\left(x_{1}, x_{2}, x_{3}\right) \\
& +t_{2} R^{0, \Lambda^{2}, \Lambda^{2}}\left(x_{1}, x_{2}, x_{3}\right) R^{\Lambda^{2}, \Lambda^{1}, \Lambda^{1}}\left(x_{1}, x_{2}, x_{3}\right) R^{\Lambda^{2}, \Lambda^{3}, \Lambda^{3}}\left(x_{1}, x_{2}, x_{3}\right) \\
& +u_{2} R^{\Lambda^{2}, \Lambda^{2}, 0}\left(x_{1}, x_{2}, x_{3}\right) R^{\Lambda^{2}, 0, \Lambda^{2}}\left(x_{1}, x_{2}, x_{3}\right) R^{0, \Lambda^{3}, \Lambda^{1}}\left(x_{1}, x_{2}, x_{3}\right) R^{0, \Lambda^{1}, \Lambda^{3}}\left(x_{1}, x_{2}, x_{3}\right) \\
0 & =s_{3} R^{\Lambda^{1}+\Lambda^{3}, \Lambda^{2}, \Lambda^{2}}\left(x_{1}, x_{2}, x_{3}\right) R^{\Lambda^{2}, \Lambda^{2}, \Lambda^{1}+\Lambda^{3}}\left(x_{1}, x_{2}, x_{3}\right) \\
& +t_{3} R^{\Lambda^{2}, 0, \Lambda^{2}}\left(x_{1}, x_{2}, x_{3}\right) R^{\Lambda^{1}, \Lambda^{2}, \Lambda^{1}}\left(x_{1}, x_{2}, x_{3}\right) R^{\Lambda^{3}, \Lambda^{2}, \Lambda^{3}}\left(x_{1}, x_{2}, x_{3}\right) \\
& +u_{3} R^{0, \Lambda^{2}, \Lambda^{2}}\left(x_{1}, x_{2}, x_{3}\right) R^{\Lambda^{2}, \Lambda^{2}, 0}\left(x_{1}, x_{2}, x_{3}\right) R^{\Lambda^{3}, 0, \Lambda^{1}}\left(x_{1}, x_{2}, x_{3}\right) R^{\Lambda^{1}, 0, \Lambda^{3}}\left(x_{1}, x_{2}, x_{3}\right) \\
0 & =s_{4} R^{\Lambda^{1}+\Lambda^{3}, \Lambda^{2}, \Lambda^{2}}\left(x_{1}, x_{2}, x_{3}\right) R^{\Lambda^{2}, \Lambda^{3}, \Lambda^{3}}\left(x_{1}, x_{2}, x_{3}\right)
\end{aligned}
$$




$$
\begin{aligned}
& +t_{4} R^{\Lambda^{1}, 0, \Lambda^{3}}\left(x_{1}, x_{2}, x_{3}\right) R^{\Lambda^{2}, \Lambda^{2}, 0}\left(x_{1}, x_{2}, x_{3}\right) R^{\Lambda^{3}, \Lambda^{3}, \Lambda^{2}}\left(x_{1}, x_{2}, x_{3}\right) \\
& +u_{4} R^{\Lambda^{1}, \Lambda^{3}, 0}\left(x_{1}, x_{2}, x_{3}\right) R^{\Lambda^{2}, 0, \Lambda^{2}}\left(x_{1}, x_{2}, x_{3}\right) R^{\Lambda^{3}, \Lambda^{2}, \Lambda^{3}}\left(x_{1}, x_{2}, x_{3}\right) \\
& 0=s_{5} R^{\Lambda^{2}, \Lambda^{1}+\Lambda^{3}, \Lambda^{2}}\left(x_{1}, x_{2}, x_{3}\right) R^{\Lambda^{3}, \Lambda^{2}, \Lambda^{3}}\left(x_{1}, x_{2}, x_{3}\right) \\
& +t_{5} R^{\Lambda^{3}, \Lambda^{1}, 0}\left(x_{1}, x_{2}, x_{3}\right) R^{0, \Lambda^{2}, \Lambda^{2}}\left(x_{1}, x_{2}, x_{3}\right) R^{\Lambda^{2}, \Lambda^{3}, \Lambda^{3}}\left(x_{1}, x_{2}, x_{3}\right) \\
& +u_{5} R^{0, \Lambda^{1}, \Lambda^{3}}\left(x_{1}, x_{2}, x_{3}\right) R^{\Lambda^{2}, \Lambda^{2}, 0}\left(x_{1}, x_{2}, x_{3}\right) R^{\Lambda^{3}, \Lambda^{3}, \Lambda^{2}}\left(x_{1}, x_{2}, x_{3}\right) \\
& 0=s_{6} R^{\Lambda^{2}, \Lambda^{2}, \Lambda^{1}+\Lambda^{3}}\left(x_{1}, x_{2}, x_{3}\right) R^{\Lambda^{3}, \Lambda^{3}, \Lambda^{2}}\left(x_{1}, x_{2}, x_{3}\right) \\
& +t_{6} R^{0, \Lambda^{3}, \Lambda^{1}}\left(x_{1}, x_{2}, x_{3}\right) R^{\Lambda^{2}, 0, \Lambda^{2}}\left(x_{1}, x_{2}, x_{3}\right) R^{\Lambda^{3}, \Lambda^{2}, \Lambda^{3}}\left(x_{1}, x_{2}, x_{3}\right) \\
& +u_{6} R^{\Lambda^{3}, 0, \Lambda^{1}}\left(x_{1}, x_{2}, x_{3}\right) R^{0, \Lambda^{2}, \Lambda^{2}}\left(x_{1}, x_{2}, x_{3}\right) R^{\Lambda^{2}, \Lambda^{3}, \Lambda^{3}}\left(x_{1}, x_{2}, x_{3}\right) \\
& 0=s_{7} R^{\Lambda^{2}, \Lambda^{2}, \Lambda^{1}+\Lambda^{3}}\left(x_{1}, x_{2}, x_{3}\right) R^{\Lambda^{1}, \Lambda^{1}, \Lambda^{2}}\left(x_{1}, x_{2}, x_{3}\right) \\
& +t_{7} R^{\Lambda^{1}, 0, \Lambda^{3}}\left(x_{1}, x_{2}, x_{3}\right) R^{0, \Lambda^{2}, \Lambda^{2}}\left(x_{1}, x_{2}, x_{3}\right) R^{\Lambda^{2}, \Lambda^{1}, \Lambda^{1}}\left(x_{1}, x_{2}, x_{3}\right) \\
& +u_{7} R^{0, \Lambda^{1}, \Lambda^{3}}\left(x_{1}, x_{2}, x_{3}\right) R^{\Lambda^{2}, 0, \Lambda^{2}}\left(x_{1}, x_{2}, x_{3}\right) R^{\Lambda^{1}, \Lambda^{2}, \Lambda^{1}}\left(x_{1}, x_{2}, x_{3}\right) \\
& 0=s_{8} R^{\Lambda^{2}, \Lambda^{1}+\Lambda^{3}, \Lambda^{2}}\left(x_{1}, x_{2}, x_{3}\right) R^{\Lambda^{1}, \Lambda^{2}, \Lambda^{1}}\left(x_{1}, x_{2}, x_{3}\right) \\
& +t_{8} R^{0, \Lambda^{3}, \Lambda^{1}}\left(x_{1}, x_{2}, x_{3}\right) R^{\Lambda^{2}, \Lambda^{2}, 0}\left(x_{1}, x_{2}, x_{3}\right) R^{\Lambda^{1}, \Lambda^{1}, \Lambda^{2}}\left(x_{1}, x_{2}, x_{3}\right) \\
& +u_{8} R^{\Lambda^{1}, \Lambda^{3}, 0}\left(x_{1}, x_{2}, x_{3}\right) R^{0, \Lambda^{2}, \Lambda^{2}}\left(x_{1}, x_{2}, x_{3}\right) R^{\Lambda^{2}, \Lambda^{1}, \Lambda^{1}}\left(x_{1}, x_{2}, x_{3}\right) \\
& 0=s_{9} R^{\Lambda^{1}+\Lambda^{3}, \Lambda^{2}, \Lambda^{2}}\left(x_{1}, x_{2}, x_{3}\right) R^{\Lambda^{2}, \Lambda^{1}, \Lambda^{1}}\left(x_{1}, x_{2}, x_{3}\right) \\
& +t_{9} R^{\Lambda^{3}, \Lambda^{1}, 0}\left(x_{1}, x_{2}, x_{3}\right) R^{\Lambda^{2}, 0, \Lambda^{2}}\left(x_{1}, x_{2}, x_{3}\right) R^{\Lambda^{1}, \Lambda^{2}, \Lambda^{1}}\left(x_{1}, x_{2}, x_{3}\right) \\
& +u_{9} R^{\Lambda^{3}, 0, \Lambda^{1}}\left(x_{1}, x_{2}, x_{3}\right) R^{\Lambda^{2}, \Lambda^{2}, 0}\left(x_{1}, x_{2}, x_{3}\right) R^{\Lambda^{1}, \Lambda^{1}, \Lambda^{2}}\left(x_{1}, x_{2}, x_{3}\right) \\
& 0=s_{10} R^{\Lambda^{1}+\Lambda^{3}, \Lambda^{2}, \Lambda^{2}}\left(x_{1}, x_{2}, x_{3}\right) R^{0, \Lambda^{1}, \Lambda^{3}}\left(x_{1}, x_{2}, x_{3}\right) \\
& +t_{10} R^{\Lambda^{1}, \Lambda^{1}, \Lambda^{2}}\left(x_{1}, x_{2}, x_{3}\right) R^{\Lambda^{3}, \Lambda^{2}, \Lambda^{3}}\left(x_{1}, x_{2}, x_{3}\right) \\
& +u_{10} R^{\Lambda^{1}, 0, \Lambda^{3}}\left(x_{1}, x_{2}, x_{3}\right) R^{\Lambda^{3}, \Lambda^{1}, 0}\left(x_{1}, x_{2}, x_{3}\right) R^{0, \Lambda^{2}, \Lambda^{2}}\left(x_{1}, x_{2}, x_{3}\right) \\
& 0=s_{11} R^{\Lambda^{2}, \Lambda^{2}, \Lambda^{1}+\Lambda^{3}}\left(x_{1}, x_{2}, x_{3}\right) R^{\Lambda^{1}, \Lambda^{3}, 0}\left(x_{1}, x_{2}, x_{3}\right) \\
& +t_{11} R^{\Lambda^{1}, \Lambda^{2}, \Lambda^{1}}\left(x_{1}, x_{2}, x_{3}\right) R^{\Lambda^{2}, \Lambda^{3}, \Lambda^{3}}\left(x_{1}, x_{2}, x_{3}\right) \\
& +u_{11} R^{\Lambda^{1}, 0, \Lambda^{3}}\left(x_{1}, x_{2}, x_{3}\right) R^{0, \Lambda^{3}, \Lambda^{1}}\left(x_{1}, x_{2}, x_{3}\right) R^{\Lambda^{2}, \Lambda^{2}, 0}\left(x_{1}, x_{2}, x_{3}\right) \\
& 0=s_{12} R^{\Lambda^{2}, \Lambda^{1}+\Lambda^{3}, \Lambda^{2}}\left(x_{1}, x_{2}, x_{3}\right) R^{\Lambda^{3}, 0, \Lambda^{1}}\left(x_{1}, x_{2}, x_{3}\right) \\
& +t_{12} R^{\Lambda^{2}, \Lambda^{1}, \Lambda^{1}}\left(x_{1}, x_{2}, x_{3}\right) R^{\Lambda^{3}, \Lambda^{3}, \Lambda^{2}}\left(x_{1}, x_{2}, x_{3}\right) \\
& +u_{12} R^{\Lambda^{3}, \Lambda^{1}, 0}\left(x_{1}, x_{2}, x_{3}\right) R^{0, \Lambda^{3}, \Lambda^{1}}\left(x_{1}, x_{2}, x_{3}\right) R^{\Lambda^{2}, 0, \Lambda^{2}}\left(x_{1}, x_{2}, x_{3}\right) \\
& 0=s_{13} R^{\Lambda^{2}, \Lambda^{1}+\Lambda^{3}, \Lambda^{2}}\left(x_{1}, x_{2}, x_{3}\right) R^{\Lambda^{1}, 0, \Lambda^{3}}\left(x_{1}, x_{2}, x_{3}\right) \\
& +t_{13} R^{\Lambda^{1}, \Lambda^{1}, \Lambda^{2}}\left(x_{1}, x_{2}, x_{3}\right) R^{\Lambda^{2}, \Lambda^{3}, \Lambda^{3}}\left(x_{1}, x_{2}, x_{3}\right) \\
& +u_{13} R^{0, \Lambda^{1}, \Lambda^{3}}\left(x_{1}, x_{2}, x_{3}\right) R^{\Lambda^{1}, \Lambda^{3}, 0}\left(x_{1}, x_{2}, x_{3}\right) R^{\Lambda^{2}, 0, \Lambda^{2}}\left(x_{1}, x_{2}, x_{3}\right) \\
& 0=s_{14} R^{\Lambda^{2}, \Lambda^{2}, \Lambda^{1}+\Lambda^{3}}\left(x_{1}, x_{2}, x_{3}\right) R^{\Lambda^{3}, \Lambda^{1}, 0}\left(x_{1}, x_{2}, x_{3}\right) \\
& +t_{14} R^{\Lambda^{2}, \Lambda^{1}, \Lambda^{1}}\left(x_{1}, x_{2}, x_{3}\right) R^{\Lambda^{3}, \Lambda^{2}, \Lambda^{3}}\left(x_{1}, x_{2}, x_{3}\right) \\
& +u_{14} R^{0, \Lambda^{1}, \Lambda^{3}}\left(x_{1}, x_{2}, x_{3}\right) R^{\Lambda^{3}, 0, \Lambda^{1}}\left(x_{1}, x_{2}, x_{3}\right) R^{\Lambda^{2}, \Lambda^{2}, 0}\left(x_{1}, x_{2}, x_{3}\right) \\
& 0=s_{15} R^{\Lambda^{1}+\Lambda^{3}, \Lambda^{2}, \Lambda^{2}}\left(x_{1}, x_{2}, x_{3}\right) R^{0, \Lambda^{3}, \Lambda^{1}}\left(x_{1}, x_{2}, x_{3}\right) \\
& +t_{15} R^{\Lambda^{1}, \Lambda^{2}, \Lambda^{1}}\left(x_{1}, x_{2}, x_{3}\right) R^{\Lambda^{3}, \Lambda^{3}, \Lambda^{2}}\left(x_{1}, x_{2}, x_{3}\right) \\
& +u_{15} R^{\Lambda^{1}, \Lambda^{3}, 0}\left(x_{1}, x_{2}, x_{3}\right) R^{\Lambda^{3}, 0, \Lambda^{1}}\left(x_{1}, x_{2}, x_{3}\right) R^{0, \Lambda^{2}, \Lambda^{2}}\left(x_{1}, x_{2}, x_{3}\right)
\end{aligned}
$$


The first 3 correspond to the coupling $\left(\Lambda^{1}+\Lambda^{2}+\Lambda^{3}, \Lambda^{1}+\Lambda^{2}+\Lambda^{3}, 2 \Lambda^{2}\right)$ and permutations thereof, the next 3 correspond to the coupling $\left(\Lambda^{1}+\Lambda^{2}+\Lambda^{3}, \Lambda^{2}+\Lambda^{3}, \Lambda^{2}+\Lambda^{3}\right)$ and permutations thereof, the next 3 correspond to the coupling $\left(\Lambda^{1}+\Lambda^{2}, \Lambda^{1}+\Lambda^{2}, \Lambda^{1}+\Lambda^{2}+\Lambda^{3}\right)$ and permutations thereof, whereas the last 6 correspond to the coupling $\left(\Lambda^{1}+\Lambda^{3}, \Lambda^{1}+\right.$ $\Lambda^{2}, \Lambda^{2}+\Lambda^{3}$ ) and permutations thereof. In the normalization chosen, (96), the parameters are worked out to be

$$
\begin{array}{lll}
\left(t_{1}, u_{1}\right)=(4,-4) & \left(t_{2}, u_{2}\right)=(-4,4) & \left(t_{3}, u_{3}\right)=(4,-4) \\
\left(t_{4}, u_{4}\right)=(2,-2) & \left(t_{5}, u_{5}\right)=(-2,2) & \left(t_{6}, u_{6}\right)=(2,2) \\
\left(t_{7}, u_{7}\right)=(-2,-2) & \left(t_{8}, u_{8}\right)=(2,2) & \left(t_{9}, u_{9}\right)=(2,-2) \\
\left(t_{10}, u_{10}\right)=(2,-2) & \left(t_{11}, u_{11}\right)=(2,2) & \left(t_{12}, u_{12}\right)=(2,2) \\
\left(t_{13}, u_{13}\right)=(-2,-2) & \left(t_{14}, u_{14}\right)=(-2,-2) & \left(t_{15}, u_{15}\right)=(-2,2)
\end{array}
$$

where we have fixed $s_{\ell}=1, \ell=1, \ldots, 15$.

\section{References}

[1] J. Rasmussen, Mod. Phys. Lett. A 13 (1998) 1213;

J. Rasmussen, Mod. Phys. Lett. A 13 (1998) 1281

[2] C.J. Cummins, M. Couture and R.T. Sharp, J. Phys. A: Math. Gen. 23 (1990) 1929

[3] L. Bégin, P. Mathieu and M.A. Walton, Mod. Phys. Lett. A 7 (1992) 3255

[4] L. Bégin, A.N. Kirillov, P. Mathieu and M.A. Walton, Lett. Math. Phys. 28 (1993) 257

[5] W. Rühl, Ann. Phys. 206 (1991) 368

[6] J. Rasmussen, Applications of Free Fields in 2D Current Algebra, Ph.D. thesis (The Niels Bohr Institute), hep-th/9610167;

J.L. Petersen, J. Rasmussen and M. Yu, Nucl. Phys. B 502 (1997) 649

[7] P. Furlan, A.Ch. Ganchev, R. Paunov and V.B. Petkova, Phys. Lett. B 267 (1991) 63 ;

P. Furlan, A.Ch. Ganchev, R. Paunov and V.B. Petkova, Nucl. Phys. B 394 (1993) 665 ;

A.Ch. Ganchev and V.B. Petkova, Phys. Lett. B 293 (1992) 56

[8] J.L. Petersen, J. Rasmussen and M. Yu, Nucl. Phys. B 457 (1995) 309

[9] P. Furlan, A.Ch. Ganchev and V.B. Petkova, Nucl. Phys. B 343 (1990) 205

[10] J. Fuchs, Affine Lie Algebras and Quantum Groups, (Cambridge Univ. Press, 1992)

[11] J. Rasmussen, Screening Currents in Affine Current Algebra, q-alg/9711017

[12] A.D. Berenstein and A.Z. Zelevinsky, J. Algebraic Combinatorics 1 (1992) 7 
[13] V.G. Kac and D.A. Kazhdan, Adv. Math. 34 (1979) 97

[14] V.G. Kac and M. Wakimoto, Proc. Natl. Acad. Sci. USA 85 (1988) 4956;

V.G. Kac, and D.A. Kazhdan, Adv. Ser. Math. Phys., Vol. 7 (World Scientific, 1989), p. 138

[15] J.L. Petersen, J. Rasmussen and M. Yu, Nucl. Phys. B 457 (1995) 343

[16] P. Furlan, A.Ch. Ganchev, R. Paunov and V.B. Petkova, in Low-Dimensional Topology and Quantum Field Theory, Ed. H. Osborn (Plenum Press, New York, 1993)

[17] J. Rasmussen, Nucl. Phys. B 510 (1998) 688 